\title{
Vibration Control of a Compressor Blade Using Position and Velocity Feedback
}

\author{
Ali Kandil and Magdy Kamel \\ Department of Physics and Engineering Mathematics, Faculty of Electronic Engineering, Menoufia University, \\ Menouf 32952, Egypt.
}

\section{(Received 20 August 2016; accepted 31 January 2017)}

Position and velocity feedback controllers are applied in this work to reduce the oscillations of a rotating blade dynamical system running at an unsteady rotating speed. Both the primary resonance and the principal parametric resonance are controlled as they are the worst cases that were verified numerically. The two modes of vibrations are found to be powerfully linearly coupled, so we have applied the controller to only one mode and the other, coupled mode follows it. The overall nonlinear behaviour of the system with and without control is investigated through the multiple time scales method. Time history and different response curves of the controlled system are included to show the controller effect.

\section{NOMENCLATURE}

$\ddot{p}, \dot{p}, p$
$\ddot{q}, \dot{q}, q$
$\mu_{1}, \mu_{2}$
$\omega$
$\beta_{11}, \beta_{21}, \beta_{13}, \beta_{22}, \beta_{5}$
$\beta_{5}$
$\beta_{14}, \beta_{24}$
$f_{0}, f$
$\Omega$
$k_{1}, k_{2}$
$\sigma_{1}, \sigma_{2}$
$\varepsilon$

Acceleration, velocity and position of the system first mode Acceleration, velocity and position of the system second mode Damping parameters of the system modes

System modes natural frequency

Coupling factors between the system modes

Cubic nonlinearity factor of the system modes

Parametric excitation parameters Constant rotating speed and magnitude of variable rotating speed

Excitation frequency

Position and velocity feedback gains

Detuning parameters Small Perturbation Parameter.

\section{INTRODUCTION}

Vibration mitigation is an extremely important goal for longer lifetimes of structures and mechanical systems. These systems suffer from nonlinear vibrations due to different reasons, for example: geometric nonlinearities, nonlinear properties of materials, and nonlinear excitation forces. In the case of enrolling such a system in a main resonance state, it may produce large amplitudes, which lead to it damaging itself, or possibly damaging the adjacent systems. One of the systems that has unwanted vibrations is the rotating beam. Rotating beams are dynamical systems that are modelled as cantilever beams with many uses in robot manipulators, helicopter blades, and compressor blades. In addition, the rotating blades may suffer from large amplitude vibrations, resulting in catastrophic results, especially when operating at high speeds, which leads to huge centrifugal force. Yoo et al. established the model for pre-twisted rotating blades and made an analysis to clar- ify the characteristics of vibration when a concentrated mass is attached to it. ${ }^{1}$ Sinha investigated the characteristic dynamics of the same model, but with a radial blade and considering a Coulomb damping with centrifugal force affecting the whole system. ${ }^{2}$ Fazelzadeh et al. adopted the differential quadrature method, first-order shear deformation theory, and Galerkin's technique to canvass on a rotating blade, which was thin-walled under a supersonic gas flow with a high temperature. ${ }^{3}$ Yao et al. utilized the Hamilton's principle and isotropic constitutive law to conclude the governing equations of the beam. ${ }^{4,5}$ They analysed the dynamics of the beam at varying speeds under a supersonic gas flow and a high temperature, considering the internal resonances $1: 1$ and $2: 1$, respectively. Theoretical and experimental investigations of the rotating blades response are conducted to eliminate or suppress the vibrations that could seriously destroy the reported structure. Vadiraja and Sahasrabudhe applied macro fibre composite (MFC) actuators and sensors and adopted the higher shear deformation theory to suppress the vibrations of a rotating beam. ${ }^{6}$ Younesian and Esmailzadeh reduced the vibrations of a rotating beam by about $50 \%$ by applying an internal (time-increasing) tensile force. ${ }^{7}$ They adopted Hamilton's principle for deriving the bending and longitudinal equations of a rotating blade. Other active control techniques have been applied to nonlinear dynamical systems and were very useful in reducing the vibrations. Fey et al. applied proportional and derivative feedback on a piecewise linear beam system with a one-sided spring element (flushing) for the steady-state oscillation reduction. ${ }^{8}$ They minimized the beam midpoint transversal amplitude at the primary resonance in a larger bandwidth of excitation. Warminski et al. analysed the use of suggested control algorithms to suppress the vibrations of a nonlinear composite beam and one of those algorithms was the position controller, which was terrific in reducing the vibrations. ${ }^{9}$ Muhammad et al. proposed a flexible manipulator (single-link) using the strategies, proportional derivative, and active force controllers. ${ }^{10}$ Eissa et al. studied the active vibration suppression of a nonlinear dynamical system via applying proportional and derivative controllers with and without the time delay ef- 


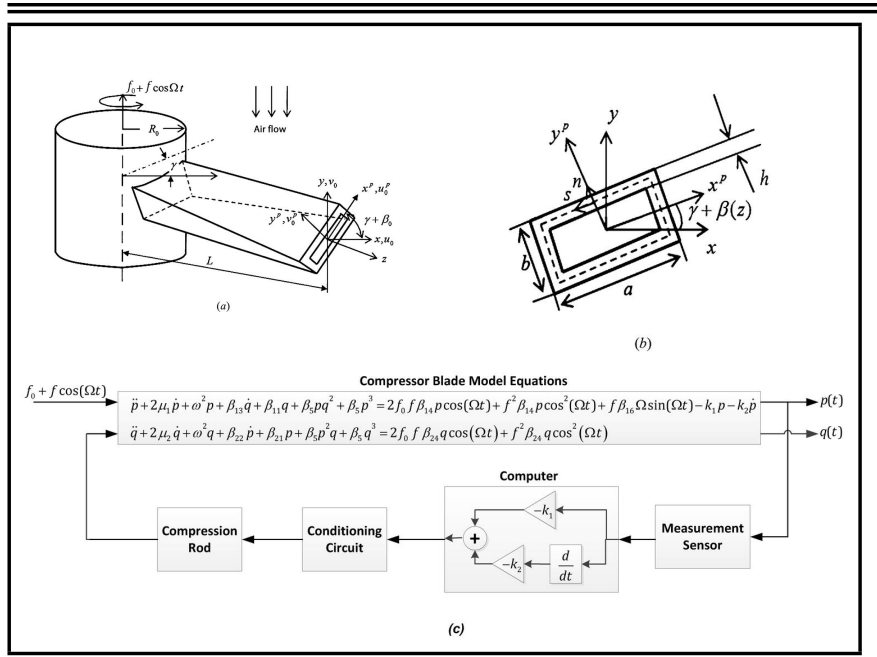

Figure 1. Rotating compressor blade model, (a) thin-walled pre-twisted blade, (b) blade cross section, and (c) block diagram of control process.

fect. ${ }^{11,12}$ They investigated the controller parameters and their effects on the output amplitude and also studied the stable region for the time delay to stay within for a better performance. In this paper, the adopted model is our case of study, as shown in Fig. 1a-b, and its derivation is included in-brief in Appendix B. ${ }^{4,5}$ The vertical and horizontal displacements of the blade cross section can be measured or sensed via sensors fixed on each blade. The measured signals will be sent back to the computer to analyse it and compute the control signal depending on it. Once the control signal is calculated, it is passed through the conditioning circuit and then it is applied on a compression rod inserted into the rotating beam's rotation axis. ${ }^{7}$ The compression rod presses the end of the blade to modify its position and reduce its vibration. The whole operation continues until the steady state amplitudes become smaller compared to those before the control. The multiple time scales method is conducted to derive the steady state equations and the results are verified by numerical simulations.

\section{MULTIPLE TIME SCALES ANALYSIS}

We have applied the control algorithm to the uncontrolled system model to have the modified equations as is follows: ${ }^{4,5}$

$$
\begin{gathered}
\ddot{p}+2 \mu_{1} \dot{p}+\omega^{2} p+\beta_{13} \dot{q}+\beta_{5} p q^{2}+\beta_{5} p^{3}= \\
2 f_{0} f \beta_{14} p \cos (\Omega t)+f^{2} \cos ^{2}(\Omega t)+f \beta_{16} \Omega \sin (\Omega t)- \\
k_{1} p-k_{2} \dot{p}, \\
\ddot{q}+2 \mu_{2} \dot{q}+\omega^{2} q+\beta_{22} \dot{p}+\beta_{21} p+\beta_{5} p^{2} q+\beta_{5} q^{3}= \\
2 f_{0} f \beta_{24} q \cos (\Omega t)+f^{2} \beta_{24} q \cos ^{2}(\Omega t) .
\end{gathered}
$$

The following parameters are suitably scaled such that:

$$
\begin{gathered}
\beta_{11}=\varepsilon \hat{\beta}_{11}, \beta_{13}=\varepsilon \hat{\beta}_{13}, \beta_{14}=\varepsilon \hat{\beta}_{14}, \beta_{16}=\varepsilon \hat{\beta}_{16}, \\
\beta_{21}=\varepsilon \hat{\beta}_{21}, \beta_{22}=\varepsilon \hat{\beta}_{22}, \beta_{24}=\varepsilon \hat{\beta}_{24}, \beta_{5}=\varepsilon \hat{\beta}_{5}, \\
k_{1}=\varepsilon \hat{k}_{1}, k_{2}=\varepsilon \hat{k}_{2}, \mu_{1}=\varepsilon \hat{\mu}_{1}, \mu_{2}=\varepsilon \hat{\mu}_{2} .
\end{gathered}
$$

Applying the multiple time scales method, an asymptotic expansion is sought as: ${ }^{13}$

$$
\begin{gathered}
p\left(T_{0}, T_{1} ; \varepsilon\right)=p_{0}\left(T_{0}, T_{1}\right)+\varepsilon p_{1}\left(T_{0}, T_{1}\right)+O\left(\varepsilon^{2}\right), \\
q\left(T_{0}, T_{1} ; \varepsilon\right)=q_{0}\left(T_{0}, T_{1}\right)+\varepsilon q_{1}\left(T_{0}, T_{1}\right)+O\left(\varepsilon^{2}\right),
\end{gathered}
$$

where $T_{0}=t, T_{1}=\varepsilon t$ are the time scales. The time derivatives will be converted to:

$$
\frac{d}{d t}=D_{0}+\varepsilon D_{1}+O\left(\varepsilon^{2}\right), \frac{d^{2}}{d t^{2}}=D_{0}^{2}+2 \varepsilon D_{1} D_{0}+O\left(\varepsilon^{2}\right),
$$

where $D_{i}=\frac{\partial}{\partial T_{i}}, i=0,1$.

Inserting Eqs. (2) to (4) into Eqs. (1) then equating like powers of $\varepsilon$ coefficients, the following are obtained:

$$
\begin{gathered}
O\left(\varepsilon^{0}\right): \\
D_{0}^{2} p_{0}+\omega^{2} p_{0}=0, \\
D_{0}^{2} q_{0}+\omega^{2} q_{0}=0 .
\end{gathered}
$$

$$
\begin{gathered}
O(\varepsilon): \\
D_{0}^{2} p_{1}+\omega^{2} p_{1}=-2 D_{1} D_{0} p_{0}-2 \hat{\mu}_{1} D_{0} p_{0}-\hat{\beta}_{13} D_{0} q_{0}- \\
\hat{\beta}_{11} q_{0}-\hat{\beta}_{5} p_{0} q_{0}^{2}-\hat{\beta}_{5} p_{0}^{3}+f_{0} f \hat{\beta}_{14} p_{0}\left(e^{i \Omega T_{0}}+e^{-i \Omega T_{0}}\right)+ \\
\frac{f^{2} \hat{\beta}_{14}}{4} p_{0}\left(e^{i \Omega T_{0}}+e^{-i \Omega T_{0}}\right)^{2}-i \frac{f \hat{\beta}_{16} \Omega}{2}\left(e^{i \Omega T_{0}}-e^{-i \Omega T_{0}}\right)- \\
\hat{k}_{1} p_{0}-\hat{k}_{2} D_{0} p_{0} \\
D_{0}^{2} q_{1}+\omega^{2} q_{1}=-2 D_{1} D_{0} q_{0}-2 \hat{\mu}_{2} D_{0} q_{0}-\hat{\beta}_{22} D_{0} p_{0}- \\
\hat{\beta}_{21} p_{0}-\hat{\beta}_{5} p_{0}^{2} q_{0}-\hat{\beta}_{5} q_{0}^{3}+f_{0} f \hat{\beta}_{24} q_{0}\left(e^{i \Omega T_{0}}+e^{-i \Omega T_{0}}\right)+ \\
\frac{f^{2} \hat{\beta}_{24}}{4} q_{0}\left(e^{i \Omega T_{0}}+e^{-i \Omega T_{0}}\right)^{2} .
\end{gathered}
$$

The complex form solutions of Eqs. 5 are expressed as is follows:

$$
\begin{aligned}
& p_{0}=A_{1} e^{i \omega T_{0}}+\bar{A}_{1} e^{-i \omega T_{0}}, \\
& q_{0}=A_{2} e^{i \omega T_{0}}+\bar{A}_{2} e^{-i \omega T_{0}},
\end{aligned}
$$

where the coefficients $A_{1}, A_{2}$ are functions of $T_{1}$ and their complex conjugates were over barred.

\subsection{Primary Resonance}

The primary resonance detuning should be represented by variable $\sigma_{1}$ in the following relation:

$$
\Omega=\omega+\sigma_{1}=\omega+\varepsilon \hat{\sigma}_{1} .
$$

Getting the solvability conditions by combining Eqs. 7 and 8 into Eqs. 6, and scaling every parameter back to its original value, we get:

$$
\begin{gathered}
-2 i \omega A_{1}-2 i \mu_{1} \omega A_{1}-\beta_{11} A_{2}-i \omega \beta_{13} A_{2}-2 \beta_{5} A_{1} A_{2} \bar{A}_{2}- \\
3 \beta_{5} A_{1}^{2} \bar{A}_{1}-\beta_{5} \bar{A}_{1} A_{2}^{2}+\frac{\beta_{14} f^{2}}{2} A_{1}+\frac{\beta_{14} f^{2}}{4} \bar{A}_{1} e^{2 i \sigma_{1} t}- \\
\frac{i}{2} \beta_{16} \Omega f e^{i \sigma_{1} t}-k_{1} A_{1}-i k_{2} \omega A_{1}=0
\end{gathered}
$$

$-2 i \omega A_{2}-2 i \mu_{2} \omega A_{2}-\beta_{21} A_{1}-i \omega \beta_{22} A_{1}-2 \beta_{5} A_{1} \bar{A}_{1} A_{2}-$

$3 \beta_{5} A_{2}^{2} \bar{A}_{2}-\beta_{5} A_{1}^{2} \bar{A}_{2}+\frac{\beta_{24} f^{2}}{2} A_{2}+\frac{\beta_{24} f^{2}}{4} \bar{A}_{2} e^{2 i \sigma_{1} t}=0$. 
Expressing $A_{1}, A_{2}$ in the polar form to be the following:

$$
\begin{gathered}
A_{1}=\frac{1}{2} a_{1} e^{i \beta_{1}} \Rightarrow \dot{A}_{1}=\frac{\dot{a}_{1}}{2} e^{i \beta_{1}}+i \frac{a_{1}}{2} \dot{\beta}_{1} e^{i \beta_{1}}, \\
A_{2}=\frac{1}{2} a_{2} e^{i \beta_{2}} \Rightarrow \dot{A}_{2}=\frac{\dot{a}_{2}}{2} e^{i \beta_{2}}+i \frac{a_{2}}{2} \dot{\beta}_{2} e^{i \beta_{2}},
\end{gathered}
$$

where $a_{m}, \beta_{m}(m=1,2)$ are amplitudes and phases of system modes.

Inserting Eqs. 10 into Eqs. 9, and after some mathematical manipulations, we get the following first-order autonomous differential equations:

$$
\begin{gathered}
\dot{a}_{1}=\mu_{1} a_{1}-\frac{\beta_{13}}{2} a_{2} \cos \phi_{2}-\frac{\beta_{5}}{8 \omega} a_{1} a_{2}^{2} \sin \left(2 \phi_{2}\right)- \\
\frac{\beta_{11}}{2 \omega} a_{2} \sin \phi_{2}+\frac{\beta_{14} f^{2}}{8 \omega} a_{1} \sin \left(2 \phi_{2}\right)-\frac{\beta_{16} \Omega f}{2 \omega} \cos \phi_{1}-\frac{k_{2}}{2} a_{1}, \\
\dot{\phi}_{1}=\sigma_{1}+\frac{\beta_{13}}{2} \frac{a_{2}}{a_{1}} \sin \phi_{2}-\frac{\beta_{5}}{8 \omega} a_{2}^{2} \cos \left(2 \phi_{2}\right)- \\
\frac{\beta_{11}}{2 \omega} \frac{a_{2}}{a_{1}} \cos \phi_{2}-\frac{\beta_{5}}{4 \omega} a_{2}^{2}-\frac{3 \beta_{5}}{8 \omega} a_{1}^{2}+\frac{\beta_{14} f^{2}}{4 \omega}+ \\
\frac{\beta_{14} f^{2}}{4 \omega} \cos \left(2 \phi_{1}\right)+\frac{\beta_{16} \Omega f}{2 \omega} \frac{1}{a_{1}} \sin \phi_{1}-\frac{k_{1}}{2 \omega}, \\
\frac{\dot{a}_{2}}{=} \mu_{2} a_{2}-\frac{\beta_{22}}{2} a_{1} \cos \phi_{2}-\frac{\beta_{5}}{8 \omega} a_{1}^{2} a_{2} \sin \left(2 \phi_{2}\right)+ \\
\frac{\beta_{21}}{2 \omega} a_{1} \sin \phi_{2}+\frac{\beta_{24} f^{2}}{8 \omega} a_{2} \sin \left(2 \phi_{1}-2 \phi_{2}\right), \\
\frac{\beta_{5}}{8 \omega} a_{1}^{2} \cos \left(2 \phi_{2}\right)+\frac{3 \beta_{5}}{8 \omega} a_{2}^{2}-\frac{\beta_{24} f^{2}}{8 \omega} \frac{\beta_{14}}{a_{1}} \cos \left(2 \phi_{1}-2 \phi_{2}\right)-\frac{\beta_{24} f^{2}}{4 \omega}+ \\
\sin \phi_{2}-\frac{\beta_{11}}{2 \omega} \frac{a_{2}}{a_{1}} \cos \left(2 \phi_{1}\right)+\frac{\beta_{16} \Omega f}{2 \omega} \frac{1}{a_{2}} \sin \phi_{2} \sin \phi_{1}+\frac{\beta_{14} f^{2}}{4 \omega}-\frac{k_{1}}{2 \omega}, \\
a_{21}
\end{gathered}
$$

where:

$$
\left\{\begin{array}{ccc}
\phi_{1}=\sigma_{1} t-\beta_{1} & \Rightarrow & \dot{\phi}_{1}=\sigma_{1}-\dot{\beta}_{1} \\
\phi_{2}=\beta_{2}-\beta_{1} & \Rightarrow & \dot{\phi}_{2}=\dot{\beta}_{2}-\dot{\beta}_{1} .
\end{array}\right\}
$$

Putting $\dot{a}_{1}=\dot{a}_{2}=\dot{\phi}_{1}=\dot{\phi}_{2}=0$ into Eqs. 11 to get the steady state equations as is follows:

$$
\begin{gathered}
\mu_{1} a_{1}=-\frac{\beta_{13}}{2} a_{2} \cos \phi_{2}-\frac{\beta_{11}}{2 \omega} a_{2} \sin \phi_{2}-\frac{\beta_{5}}{8 \omega} a_{1} a_{2}^{2} \sin \left(2 \phi_{2}\right)+ \\
\frac{\beta_{14} f^{2}}{8 \omega} a_{1} \sin \left(2 \phi_{1}\right)-\frac{\beta_{16} \Omega f}{2 \omega} \cos \phi_{1}-\frac{k_{2}}{2} a_{1} \\
-\sigma_{1} a_{1}=\frac{\beta_{13}}{2} a_{2} \sin \phi_{2}-\frac{\beta_{11}}{2 \omega} a_{2} \cos \phi_{2}-\frac{\beta_{5}}{4 \omega} a_{1} a_{2}^{2}- \\
\frac{\beta_{5}}{8 \omega} a_{1} a_{2}^{2} \cos \left(2 \phi_{2}\right)-\frac{3 \beta_{5}}{8 \omega} a_{1}^{3}+\frac{\beta_{14} f^{2}}{8 \omega} a_{1} \cos \left(2 \phi_{1}\right)+ \\
\frac{\beta_{16} \Omega f}{2 \omega} \sin \phi_{1}+\frac{\beta_{14} f^{2}}{4 \omega} a_{1}-\frac{k_{1}}{2 \omega}
\end{gathered}
$$

$\mu_{2} a_{2}=-\frac{\beta_{22}}{2} a_{1} \cos \phi_{2}+\frac{\beta_{21}}{2 \omega} a_{1} \sin \phi_{2}+\frac{\beta_{5}}{8 \omega} a_{1}^{2} a_{2} \sin \left(2 \phi_{2}\right)+$

$$
\frac{\beta_{24} f^{2}}{8 \omega} a_{2} \sin \left(2 \phi_{1}-2 \phi_{2}\right)
$$$$
\sigma_{1} a_{1}=\frac{\beta_{22}}{2} a_{1} \sin \phi_{2}+\frac{\beta_{21}}{2 \omega} a_{1} \cos \phi_{2}+\frac{\beta_{5}}{4 \omega} a_{1}^{2} a_{2}+
$$$$
\frac{\beta_{5}}{8 \omega} a_{1}^{2} a_{2} \cos \left(2 \phi_{2}\right)+\frac{3 \beta_{5}}{8 \omega} a_{2}^{3}-\frac{\beta_{24} f^{2}}{8 \omega} a_{2} \cos \left(2 \phi_{1}-2 \phi_{2}\right)-
$$

$$
\frac{\beta_{24} f^{2}}{4 \omega} a_{2} \text {. }
$$

Equations 2.1 are solved simultaneously by applying mathematical continuation methods via MATLAB software for obtaining the steady state amplitudes and phases. The stability of these solutions is analysed by the Lyapunov first method as is follows:

$$
\left(\begin{array}{c}
\dot{a}_{1} \\
\dot{\phi}_{1} \\
\dot{a}_{2} \\
\dot{\phi}_{2}
\end{array}\right)=\left(\begin{array}{llll}
v_{11} & v_{12} & v_{13} & v_{14} \\
v_{21} & v_{22} & v_{23} & v_{24} \\
v_{31} & v_{32} & v_{33} & v_{34} \\
v_{41} & v_{42} & v_{43} & v_{44}
\end{array}\right)\left(\begin{array}{c}
a_{1} \\
\phi_{1} \\
a_{2} \\
\phi_{2}
\end{array}\right),
$$

where the entries $v_{i j}\{i, j=1,2,3,4\}$ are included in Appendix A.

\subsection{Principal Parametric Resonance}

The principal parametric resonance detuning should be depicted by the variable $\sigma_{2}$ into the following relation:

$$
\Omega=2 \omega+\sigma_{2}=2 \omega+\varepsilon \hat{\sigma_{2}}
$$

Similarly, as in subsection 2.1, we get:

$$
\begin{gathered}
-2 i \omega A_{1}-2 i \mu_{1} \omega A_{1}-i \omega \beta_{13} A_{2}-\beta_{11} A_{2}-2 \beta_{5} A_{1} A_{2} \bar{A}_{2}- \\
\beta_{5} \bar{A}_{1} A_{2}^{2}-3 \beta_{5} A_{1}^{2} \bar{A}_{1}+\frac{\beta_{14} f^{2}}{2} A_{1}+f_{0} f \beta_{14} \bar{A}_{1} e^{i \sigma_{2} t}- \\
k_{1} A_{1}-i k_{2} \omega A_{1}=0, \\
-2 i \omega A_{2}-2 i \mu_{2} \omega A_{2}-i \omega \beta_{22} A_{1}-\beta_{21} A_{1}-2 \beta_{5} A_{1} \bar{A}_{1} A_{2}- \\
\beta_{5} A_{1}^{2} \bar{A}_{2}-3 \beta_{5} A_{2}^{2} \bar{A}_{2}+\frac{\beta_{24} f^{2}}{2} A_{2}+f_{0} f \beta_{24} \bar{A}_{2} e^{i \sigma_{2} t}=0 .
\end{gathered}
$$


Substituting the quantities $A_{1}, A_{2}$ from Eqs. 10 into Eqs. 2.2, and after some mathematical manipulations, we get the following first-order autonomous differential equations:

$$
\begin{gathered}
\dot{a}_{1}=-\mu_{1} a_{1}-\frac{\beta_{13}}{2} a_{2} \cos \phi_{2}-\frac{\beta_{11}}{2 \omega} a_{2} \sin \phi_{2}- \\
\frac{\beta_{5}}{8 \omega} a_{1} a_{2}^{2} \sin \left(2 \phi_{2}\right)+\frac{f_{0} f \beta_{14}}{2 \omega} a_{1} \sin \phi_{1}-\frac{k_{2}}{2} a_{1}, \\
\dot{\phi}_{1}=\sigma_{2}+\beta_{13} \frac{a_{2}}{a_{1}} \sin \phi_{2}-\frac{\beta_{11}}{\omega} \frac{a_{2}}{a_{1}} \cos \phi_{2}-\frac{\beta_{5}}{2 \omega} a_{2}^{2}- \\
\frac{\beta_{5}}{4 \omega} a_{2}^{2} \cos \left(2 \phi_{2}\right)-\frac{3 \beta_{5}}{4 \omega} a_{1}^{2}+\frac{\beta_{14} f^{2}}{2 \omega}+\frac{f_{0} f \beta_{14}}{\omega} \cos \phi_{1}-\frac{k_{1}}{\omega}, \\
\dot{a}_{2}=-\mu_{2} a_{2}-\frac{\beta_{22}}{2} a_{1} \cos \phi_{2}+\frac{\beta_{21}}{2 \omega} a_{1} \sin \phi_{2}+ \\
\frac{\beta_{5}}{8 \omega} a_{1}^{2} a_{2} \sin \left(2 \phi_{2}\right)+\frac{f_{0} f \beta_{24}}{2 \omega} a_{2} \sin \left(\phi_{1}-2 \phi_{2}\right), \\
\dot{\phi}_{2}=\frac{\beta_{22}}{2} \frac{a_{1}}{a_{2}} \sin \phi_{2}+\frac{\beta_{21}}{2 \omega} \frac{a_{1}}{a_{2}} \cos \phi_{2}+\frac{\beta_{5}}{4 \omega} a_{1}^{2}+ \\
\frac{\beta_{5}}{8 \omega} a_{1}^{2} \cos \left(2 \phi_{2}\right)+\frac{3 \beta_{5}}{8 \omega} a_{2}^{2}-\frac{f_{0} f \beta_{24}}{2 \omega} \cos \left(\phi_{1}-2 \phi_{2}\right)- \\
\frac{\beta_{24} f^{2}}{4 \omega}+\frac{\beta_{13}}{2} \frac{a_{2}}{a_{1}} \sin \phi_{2}-\frac{\beta_{11}}{2 \omega} \frac{a_{2}}{a_{1}} \cos \phi_{2}-\frac{\beta_{5}}{4 \omega} a_{2}^{2}- \\
\left.a_{2}\right)-\frac{3 \beta_{5}}{8 \omega} a_{1}^{2}+\frac{f_{0} f \beta_{14}}{2 \omega} \cos \phi_{1}+\frac{\beta_{14} f^{2}}{4 \omega}-\frac{k_{1}}{2 \omega},
\end{gathered}
$$

where

$$
\left\{\begin{array}{ccc}
\phi_{1}=\sigma_{2} t-\beta_{1} & \Rightarrow & \dot{\phi}_{1}=\sigma_{2}-2 \dot{\beta}_{1} \\
\phi_{2}=\beta_{2}-\beta_{1} & \Rightarrow & \dot{\phi}_{2}=\dot{\beta}_{2}-\dot{\beta}_{1} .
\end{array}\right\}
$$

Putting $\dot{a}_{1}=\dot{a}_{2}=\dot{\phi}_{1}=\dot{\phi}_{2}=0$ into Eqs. 17 to get the steady state equations as is follows:

$$
\begin{gathered}
\mu_{1} a_{1}=-\frac{\beta_{13}}{2} a_{2} \cos \phi_{2}-\frac{\beta_{11}}{2 \omega} a_{2} \sin \phi_{2}- \\
\frac{\beta_{5}}{8 \omega} a_{1} a_{2}^{2} \sin \left(2 \phi_{2}\right)+\frac{f_{0} f \beta_{14}}{2 \omega} a_{1} \sin \phi_{1}-\frac{k_{2}}{2} a_{1} \\
-\frac{\sigma_{2}}{2} a_{1}=\frac{\beta_{13}}{2} a_{2} \sin \phi_{2}-\frac{\beta_{11}}{2 \omega} a_{2} \cos \phi_{2}- \\
\frac{\beta_{5}}{8 \omega} a_{1} a_{2}^{2} \cos \left(2 \phi_{2}\right)+\frac{f_{0} f \beta_{14}}{2 \omega} a_{1} \cos \phi_{1}-\frac{\beta_{5}}{4 \omega} a_{1} a_{2}^{2}- \\
\frac{3 \beta_{5}}{8 \omega} a_{1}^{3}+\frac{\beta_{14} f^{2}}{4 \omega} a_{1}-\frac{k_{1}}{2 \omega} a_{1}, \\
\mu_{2} a_{2}=-\frac{\beta_{22}}{2} a_{1} \cos \phi_{2}+\frac{\beta_{21}}{2 \omega} a_{1} \sin \phi_{2}+ \\
\frac{\beta_{5}}{8 \omega} a_{1}^{2} a_{2} \sin \left(2 \phi_{2}\right)+\frac{f_{0} f \beta_{24}}{2 \omega} a_{2} \sin \left(\phi_{1}-2 \phi_{2}\right) \\
\frac{\sigma_{2}}{2} a_{2}=\frac{\beta_{22}}{2} a_{1} \sin \phi_{2}+\frac{\beta_{21}}{2 \omega} a_{1} \cos \phi_{2}+ \\
\frac{\beta_{5}}{8 \omega} a_{1}^{2} a_{2} \cos \left(2 \phi_{2}\right)-\frac{f_{0} f \beta_{24}}{2 \omega} a_{2} \cos \left(\phi_{1}-2 \phi_{2}\right)+ \\
a_{1}^{2} a_{2}+\frac{3 \beta_{5}}{8 \omega} a_{2}^{3}-\frac{\beta_{24} f^{2}}{4 \omega} a_{2} \cdot
\end{gathered}
$$
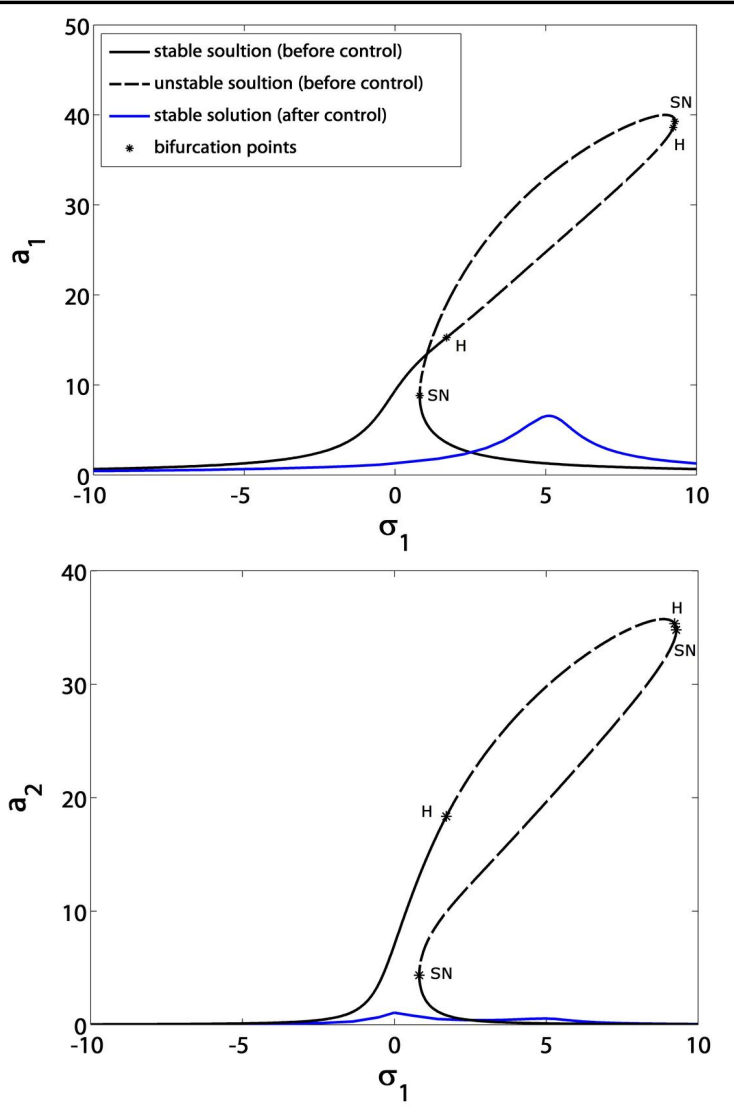

Figure 2. Frequency response curves of system modes before and after control

Equations 19 are solved simultaneously by applying mathematical continuation methods via MATLAB software for obtaining the steady state amplitudes and phases. The stability of these solutions is analysed by Lyapunov first method as is follows:

$$
\left(\begin{array}{c}
\dot{a}_{1} \\
\dot{\phi}_{1} \\
\dot{a}_{2} \\
\dot{\phi}_{2}
\end{array}\right)=\left(\begin{array}{llll}
\delta_{11} & \delta_{12} & \delta_{13} & \delta_{14} \\
\delta_{21} & \delta_{22} & \delta_{23} & \delta_{24} \\
\delta_{31} & \delta_{32} & \delta_{33} & \delta_{34} \\
\delta_{41} & \delta_{42} & \delta_{43} & \delta_{44}
\end{array}\right)\left(\begin{array}{c}
a_{1} \\
\phi_{1} \\
a_{2} \\
\phi_{2}
\end{array}\right),
$$

where the entries $\delta_{i j},\{i, j=1,2,3,4\}$ are included in Appendix A.

\section{PERTURBATION CURVES}

All the resulted perturbation curves are plotted and discussed in this section. The stable solutions are denoted by solid lines, while the unstable solutions are denoted by dashed ones. The asterisks denote the bifurcation points ( $\mathrm{SN}$ stands for Saddle-Node, H stands for Hopf, and PF stands for Pitchfork).

\subsection{Primary Resonance}

In the primary resonance case, the parameters values for plotting the curves are given by: $\mu_{1}=\mu_{2}=0.5, \Omega=$ $\omega=100, \beta_{13}=-0.82, \beta_{11}=-0,003, \beta_{14}=0.55, \beta_{5}=$ $0.9, \beta_{16}=6.55, \beta_{22}=-0.82, \beta_{21}=-0.001, \beta_{24}=$ $0.5, f_{0}=7, f=2, k_{1}=1000, k_{2}=1, \sigma_{1}=0$. In Fig. 2 , the system modes amplitudes are plotted as functions of the frequency detuning $\sigma_{1}$. It shows that the system before the control (black lines) suffers from jump phenomena, saddle node 


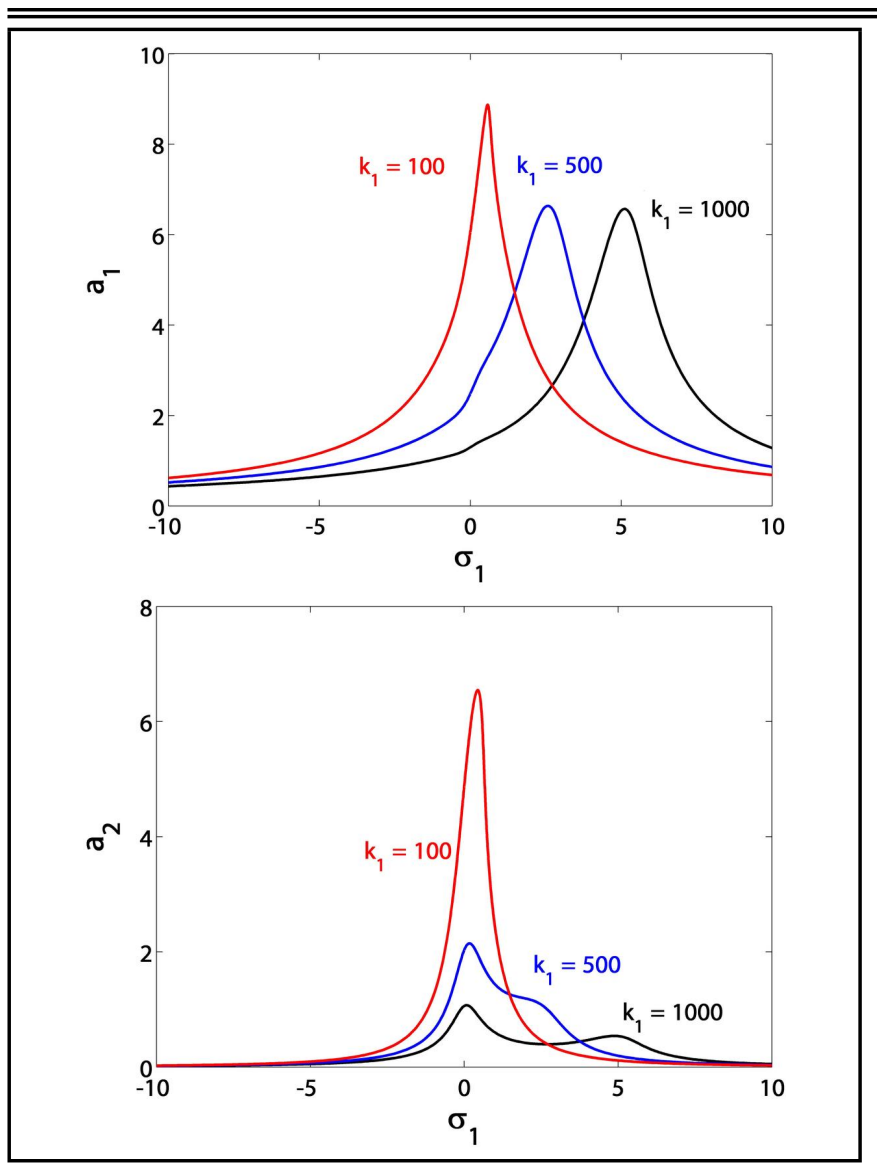

Figure 3. Various $k_{1}$ with frequency response curves

bifurcation points, and Hopf bifurcation points. After the control (blue lines), the jump phenomena, saddle-node, and Hopf bifurcations have been eliminated and the system modes amplitudes have been suppressed to low levels.

Figure 3 demonstrates the effect of varying the position feedback gain $k_{1}$ on the frequency response curves. For the system's first mode, increasing $k_{1}$ shifts the whole curve to the right because the natural frequency $\omega$ has been raised. For the system's second mode, increasing $k_{1}$ reduces the peak value and slightly raises another peak to the right of the old one.

Figure 4 demonstrates the effect of varying the velocity feedback gain $k_{2}$ on the frequency response curves. For the system's first mode, increasing $k_{2}$ suppresses the peak value, as it acts as a damping term. For the system's second mode, increasing $k_{2}$ reduces the new appeared peak value.

Figures 5 and 6 declare the response curves and response curves. It is noticeable from Fig. 5) that increasing or decreasing reduces the system's modes amplitudes with shifting the frequency response curves to the right or the left depending on the gain sign. Also, in Fig. 6, increasing reduces the system's modes amplitudes as it acts as an external damping quantity. Decreasing $k_{2}$ adds energy to the system and forces it to pass through the Hopf bifurcation to produce unstable motions.

Figure 7 depicts the output amplitudes as functions of the excitation force. It is observed that increasing the excitation force slightly (before the control) generates large output amplitudes but the relation became linear with a decreased slope (after the control).

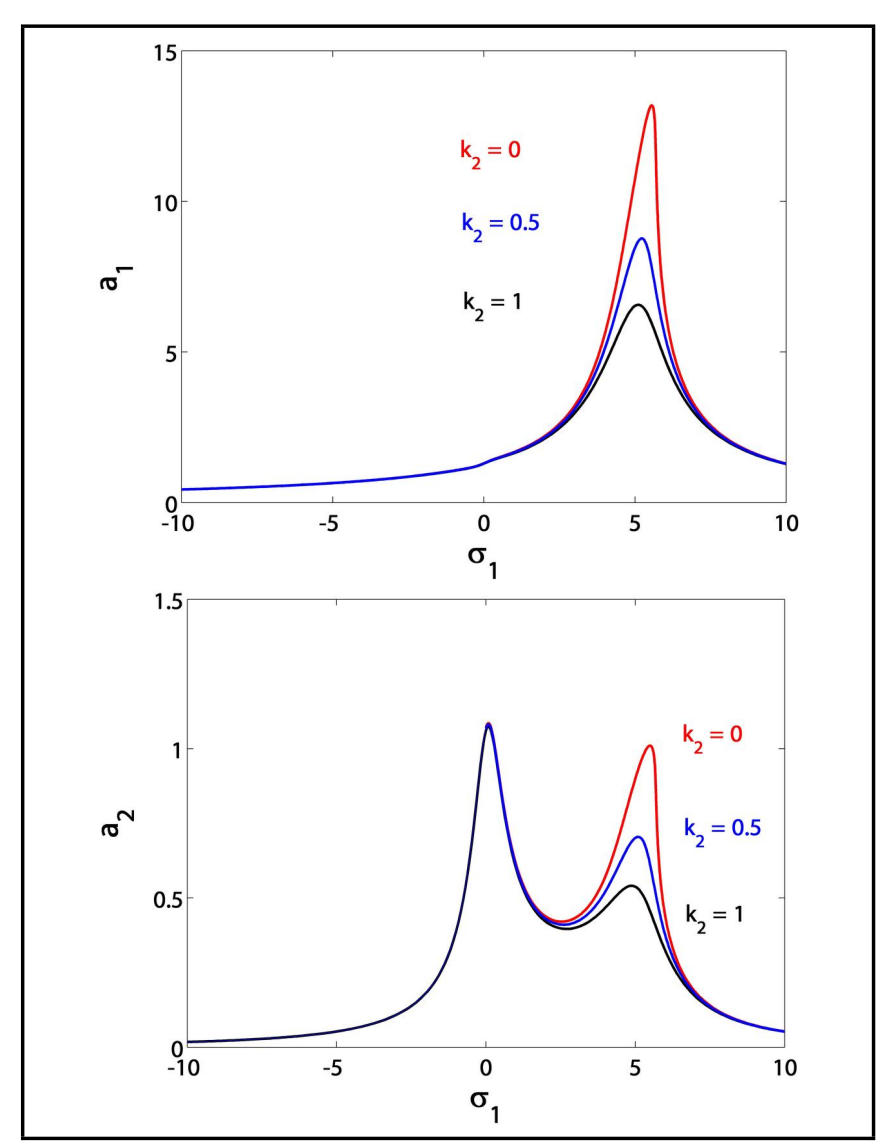

Figure 4. Various $k_{2}$ with frequency response curves
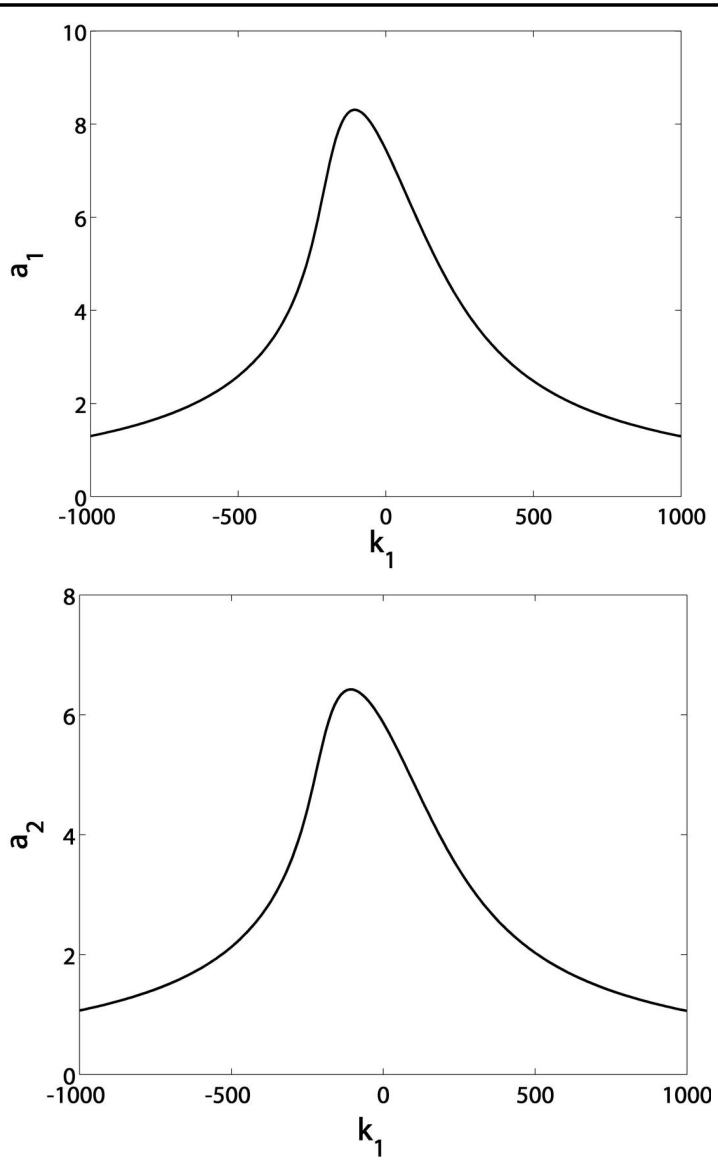

Figure 5. $k_{1}$ response curves 


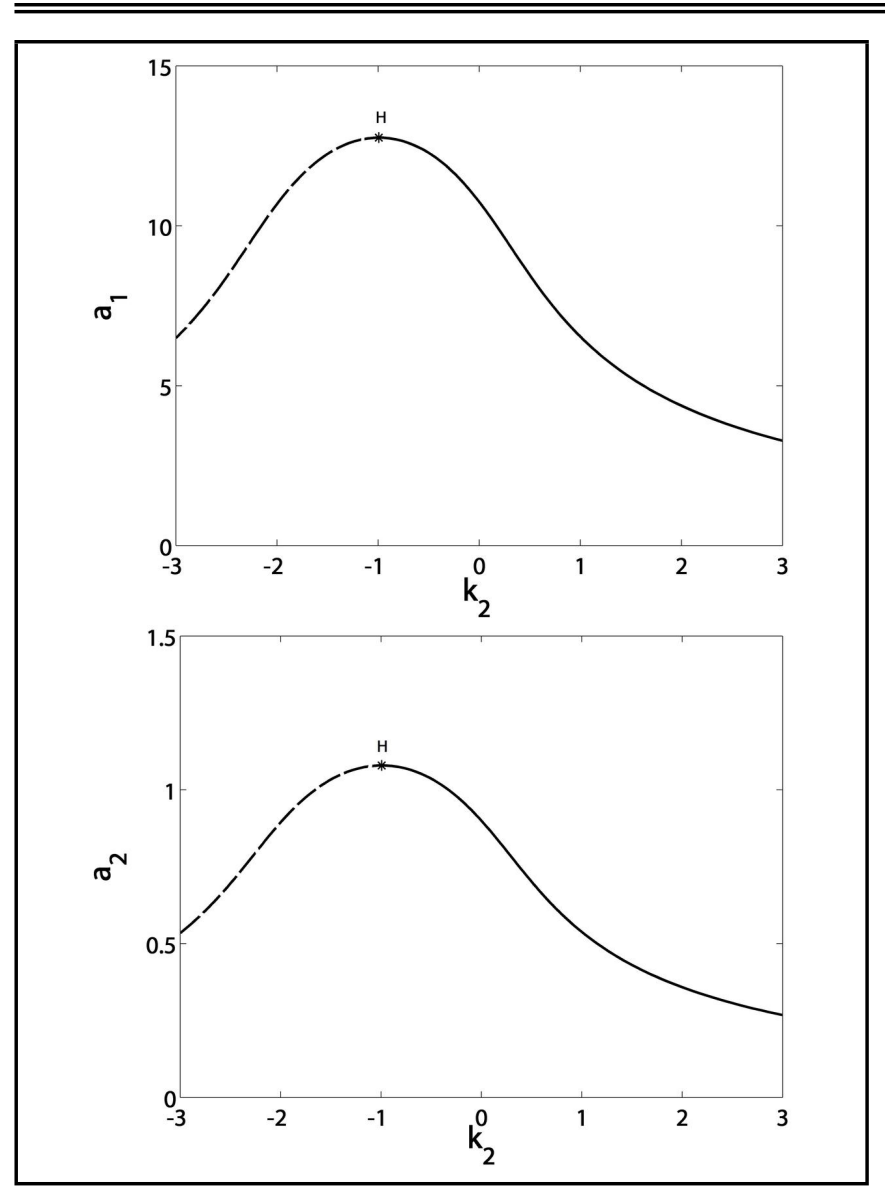

Figure 6. $k_{2}$ response curves

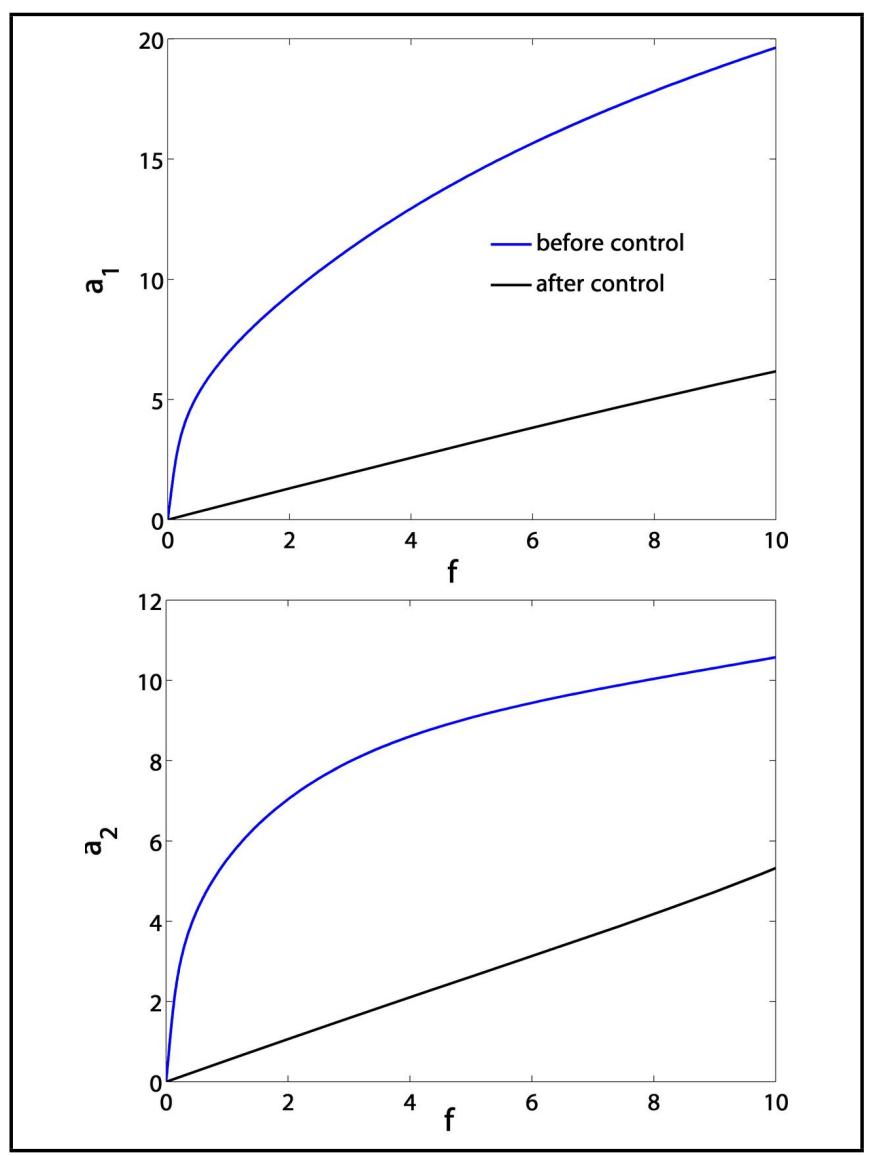

Figure 7. Force response curves at $\sigma_{1}=0$

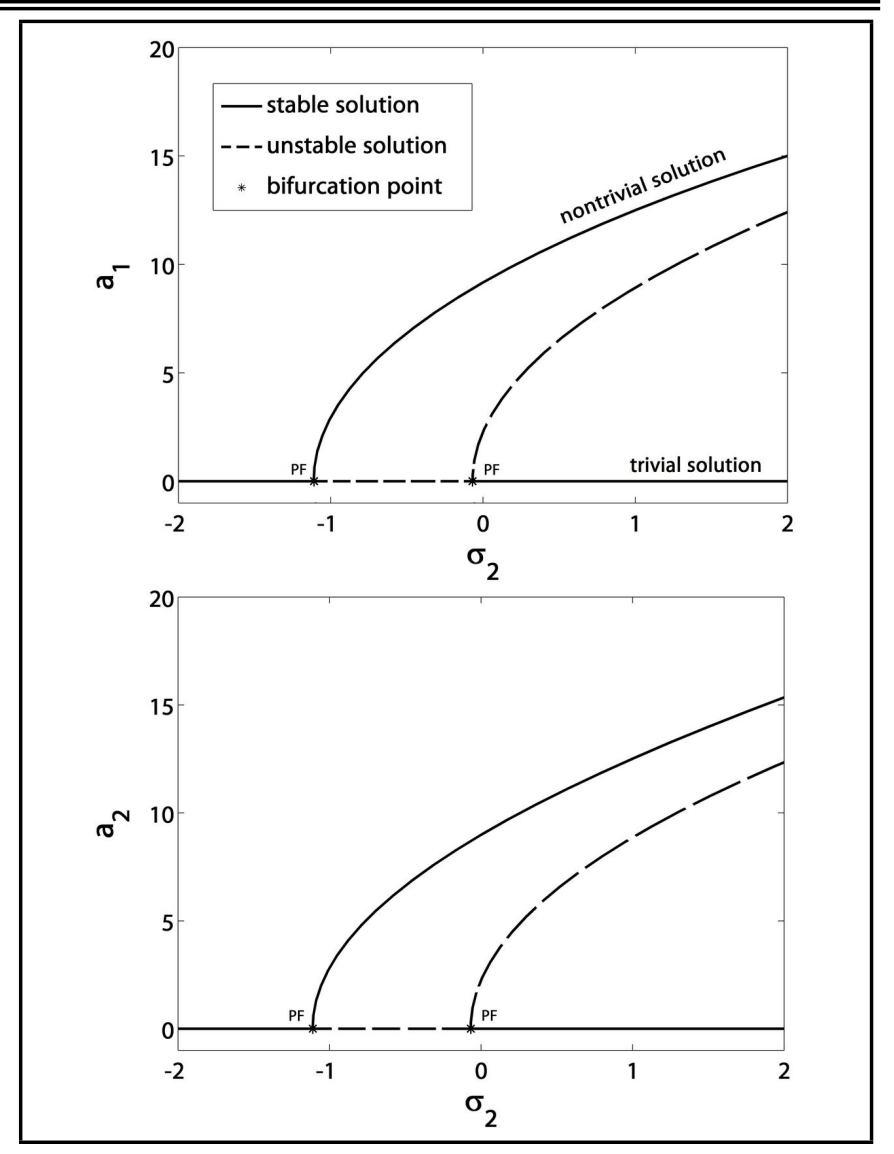

Figure 8. System modes frequency response curves before control

\subsection{Principal Parametric Resonance}

In the principal parametric resonance case, all the parameters values for plotting the curves are given by: $\mu_{1}=\mu_{2}=$ $0.5, \Omega=\omega=200, \beta_{13}=-0.82, \beta_{11}=-0,003, \beta_{14}=$ $0.55, \beta_{5}=0.9, \beta_{16}=6.55, \beta_{22}=-0.82, \beta_{21}=$ $-0.001, \beta_{24}=0.5, f_{0}=7, f=2, k_{1}=1000, k_{2}=1, \sigma_{2}=$ 0. In Fig. 7, the system's modes amplitudes are trivial (zero solution), then the frequency increases and the system passes through a super-critical pitchfork bifurcation, changing its path to the nontrivial amplitudes (non-zero solution). After reaching a given point on the nontrivial path, the system jumps down to the sub-critical pitchfork bifurcation to return back the trivial one.

After the control in Fig. 9, the controller has eliminated the two pitchfork points and remains only the trivial solution.

Figures 10 and 11 show how $k_{1}$ and $k_{2}$ affect the output amplitudes. We can see that the output amplitudes are inversely proportional to the factor $k_{1}$ in the range $-169 \leq k_{1} \leq 175$, but outside that range, the system returns to the trivial solution. In Fig. 11, $k_{2}$ is directly proportional to the first mode amplitude, but inversely proportional to the second mode amplitude in the range $-0.3 \leq k_{1} \leq 1$. For $k_{1} \geq 1$, the system returns to the trivial solution.

Figures 12 to 15 show affecting the output amplitudes with varying excitation force, with and without control. Before the control, it is clear in Fig. 12 that the output amplitudes are trivial until a given force value is reached (super-critical pitchfork), then the output amplitudes are nontrivial. In Fig. 14, a saddle-node bifurcation point faces the system when changing the initial conditions to make the system jump suddenly from 


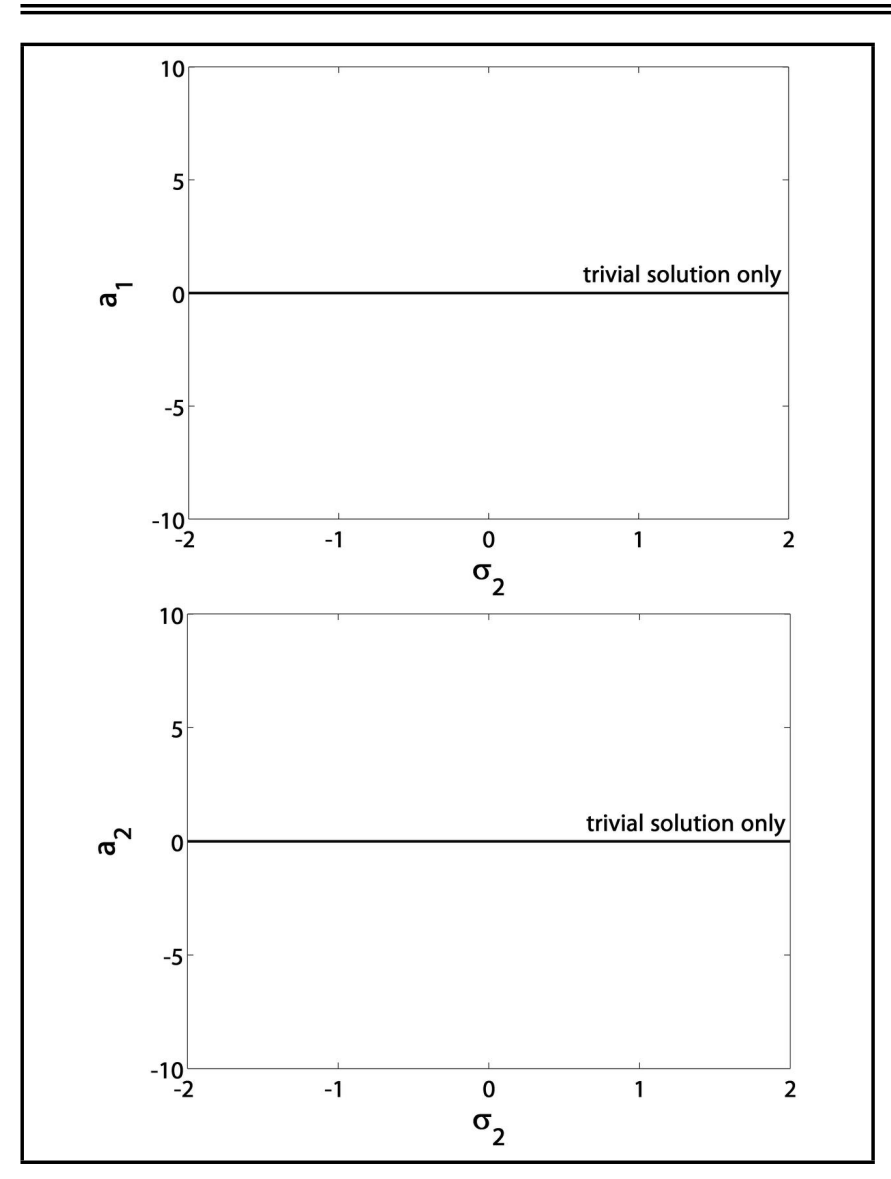

Figure 9. System modes frequency response curves after control

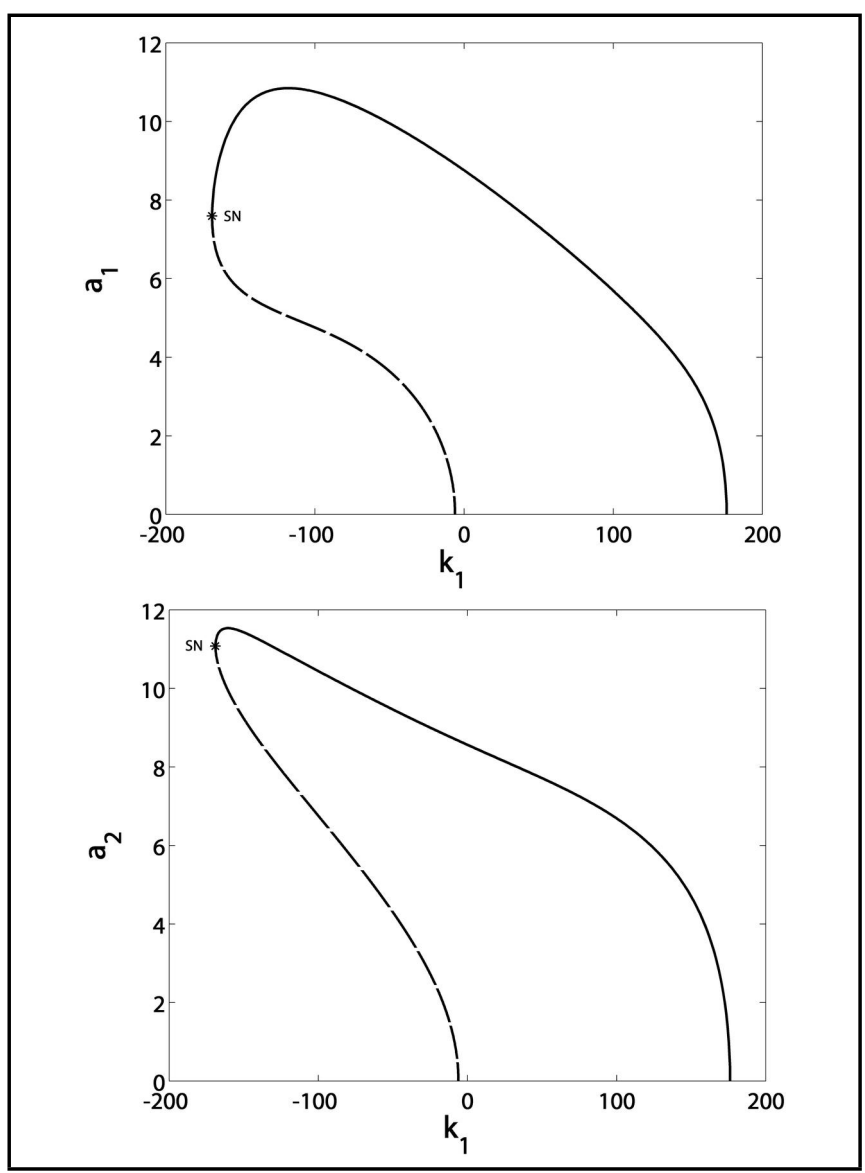

Figure 10. $k_{1}$ response curves
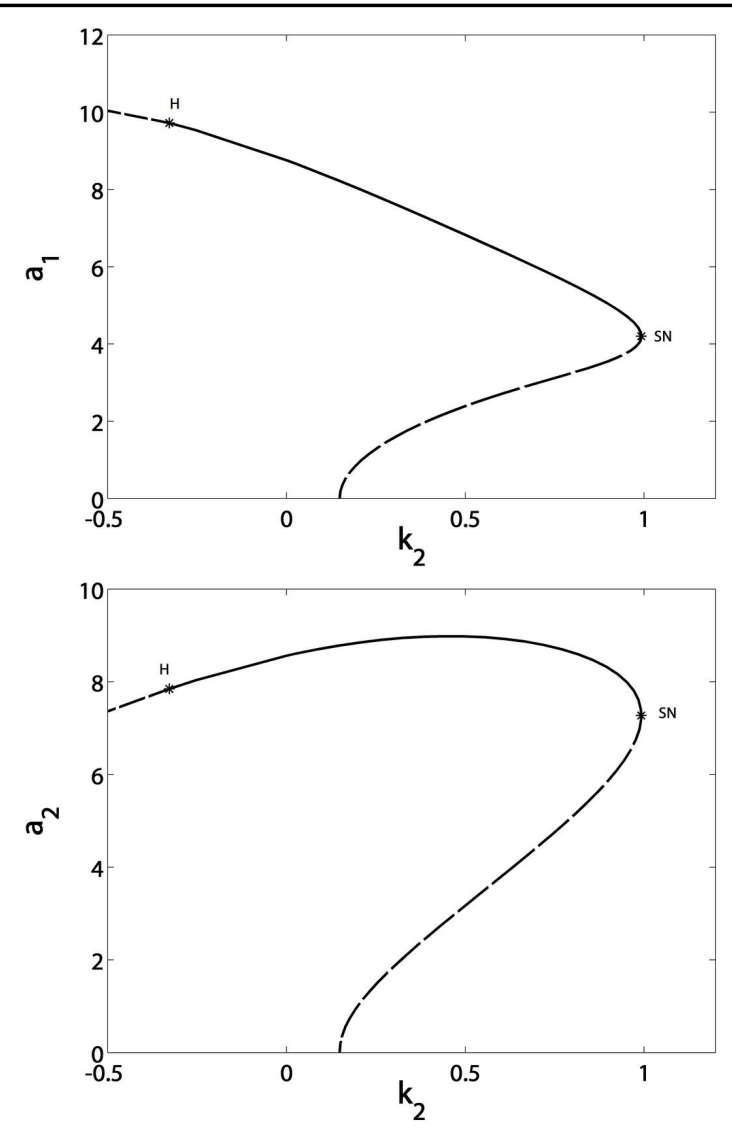

Figure 11. $k_{2}$ response curves

the trivial solution to the nontrivial one. After the control in Figs. 13 and 15, the bifurcation points vanish and the trivial output amplitudes remain.

\section{TIME HISTORY}

\subsection{Primary Resonance}

The time history before the control is shown in Fig. 16 and after the control in Fig. 17 with the same conditions (rest case). From the figures, we see that the output's steady amplitudes have been suppressed from about 9.29 and 6.96 to about 1.29 and 1.05 , respectively. The proposed controller has an effectiveness $E_{a}\left(E_{a}=\right.$ amplitude without controller / amplitude with controller) which is about 7 and 6 for the first and second modes, respectively.

\subsection{Principal Parametric Resonance}

The time history before the control is shown in Fig. 18 and after the control in Fig. 19, with the same conditions (rest case). From the figures we see that the output's steady amplitudes have been suppressed from about 8.78 and 8.49 to about 0.66 and 0.004 , respectively, to show an effectiveness $E_{a}$, which is about 13 and 2122 for the first and second modes, respectively.

\section{VALIDATION CURVES}

The validation curves are plotted using the fourth-order Runge-Kutta technique to integrate Eqs. 1 numerically. The 


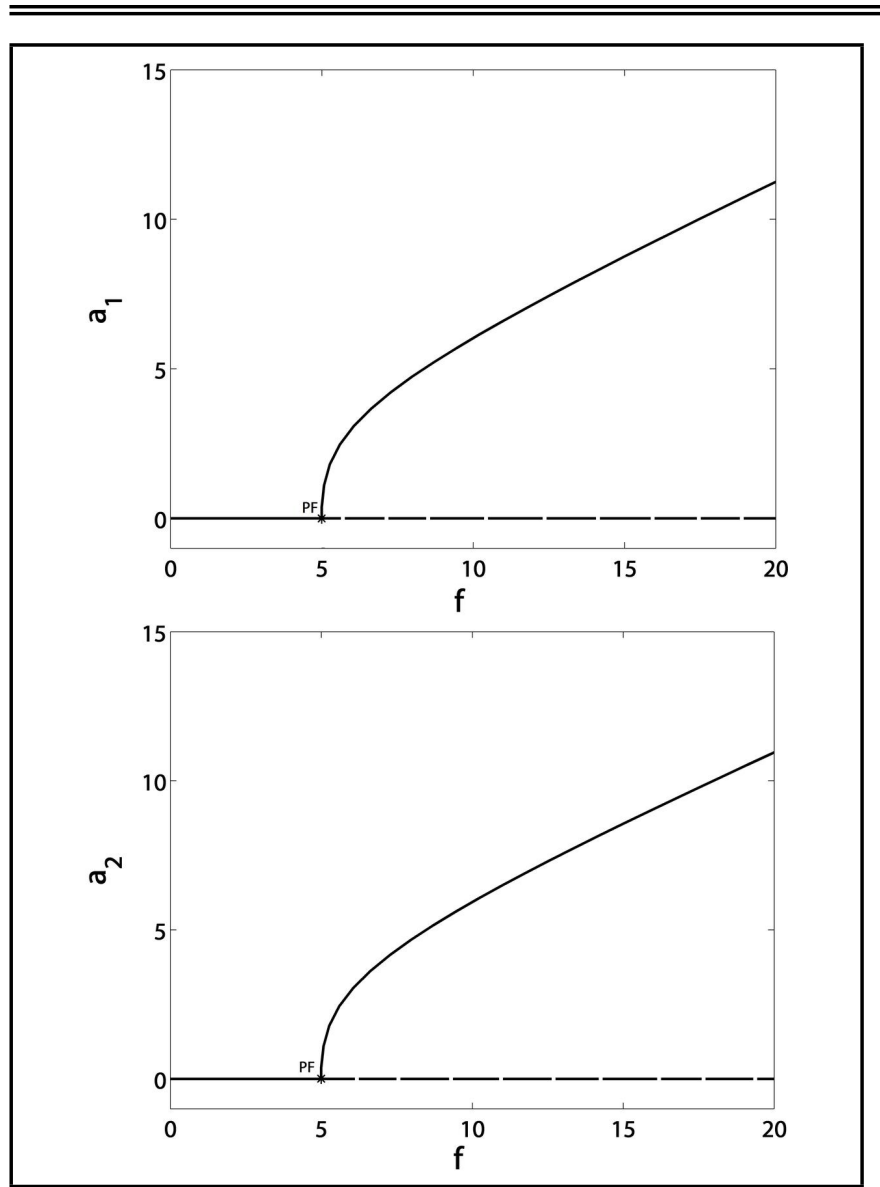

Figure 12. Force response curves at $\sigma_{2}=-0.1$ (before the control)

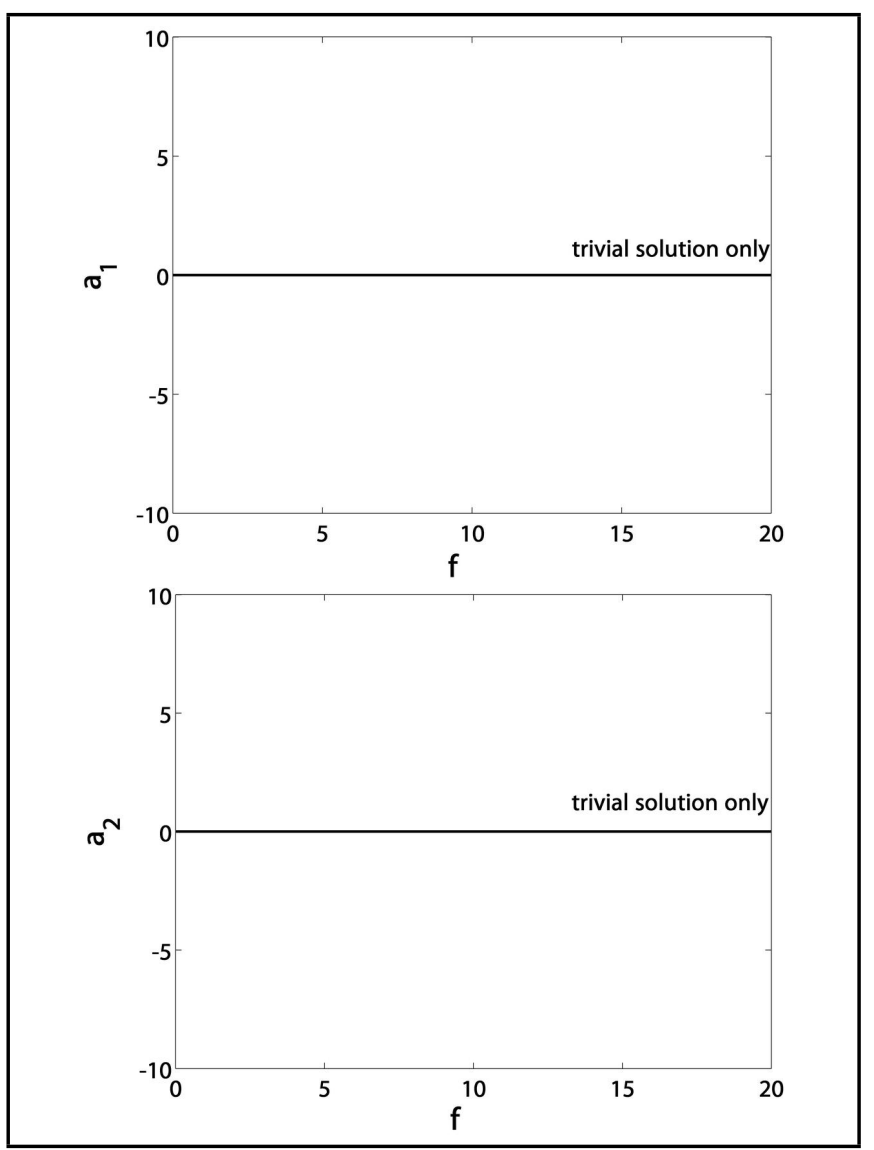

Figure 13. Force response curves at $\sigma_{2}=-0.1$ (after the control)

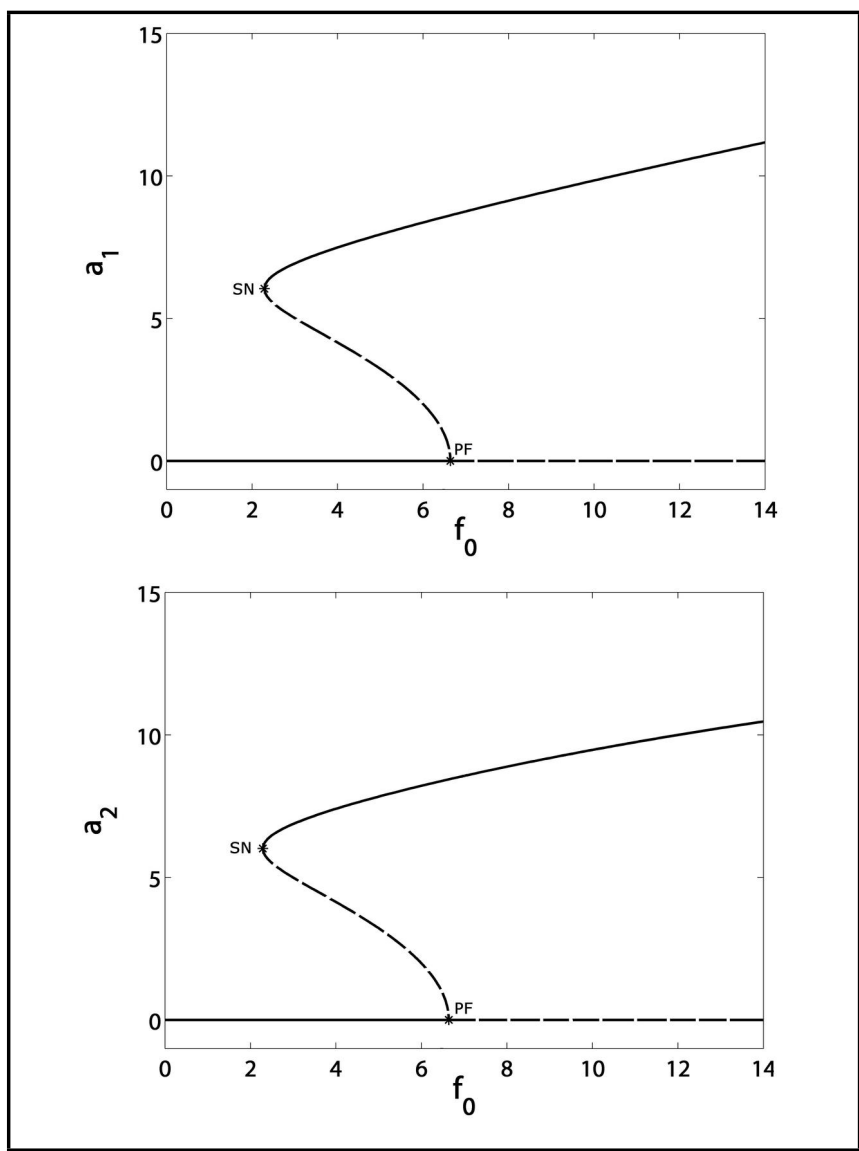

Figure 14. Force response curves at $\sigma_{2}=-0.1$ (before the control)

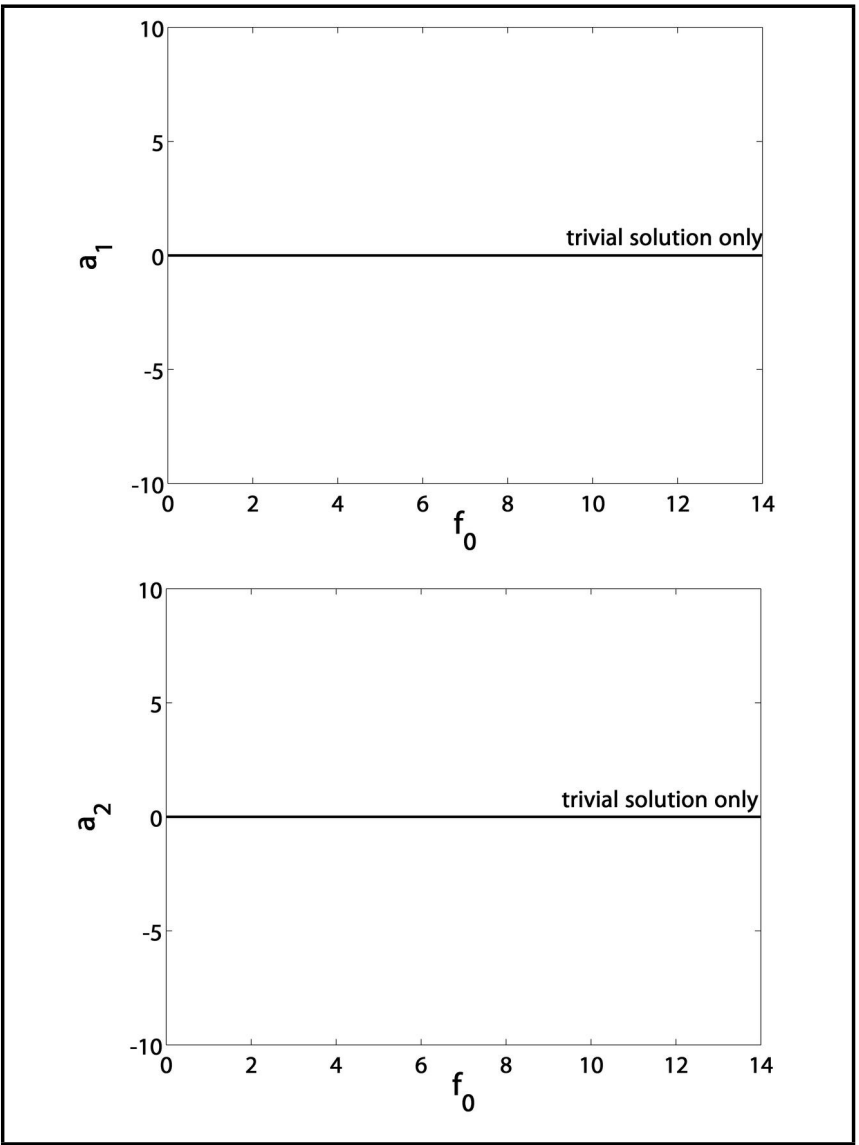

Figure 15. Force response curves at $\sigma_{2}=-0.1$ (after the control) 


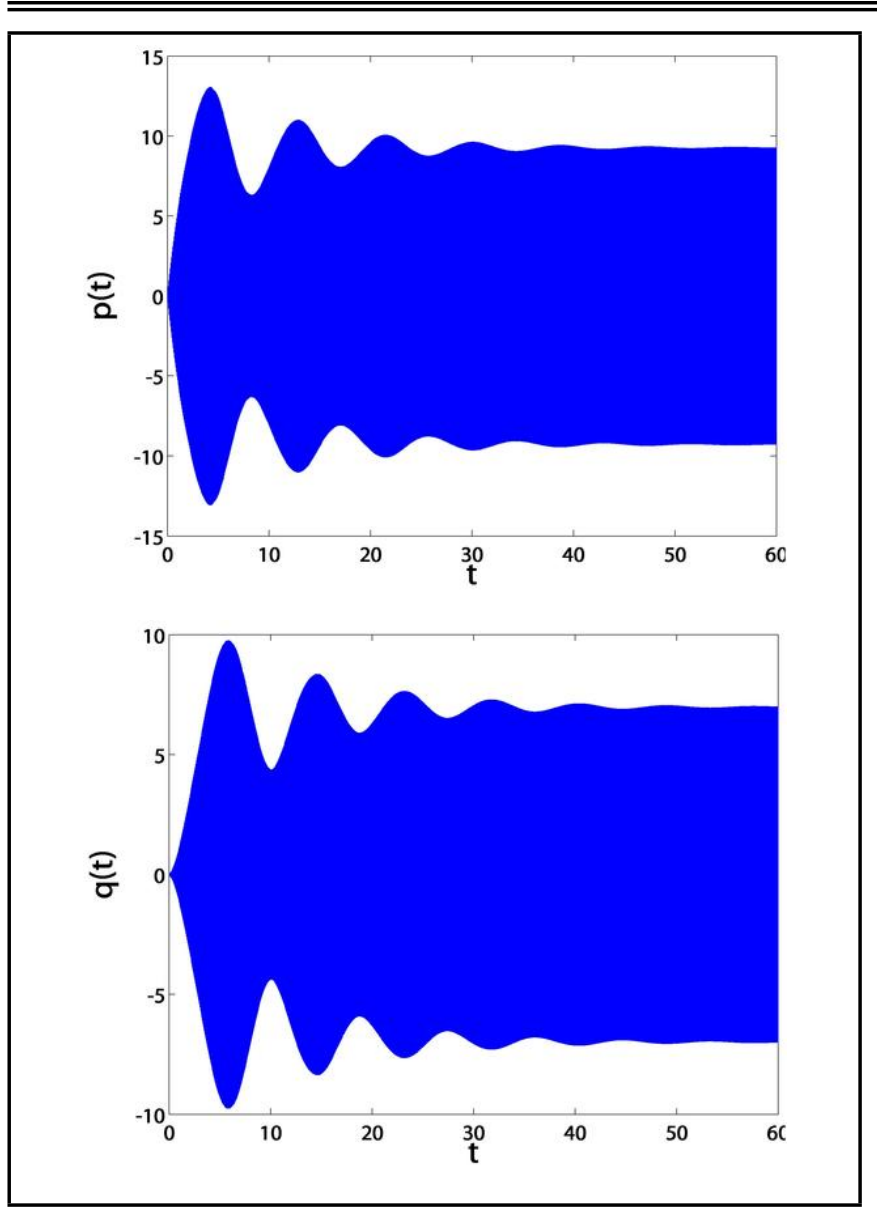

Figure 16. Time history before the control at zero initial conditions

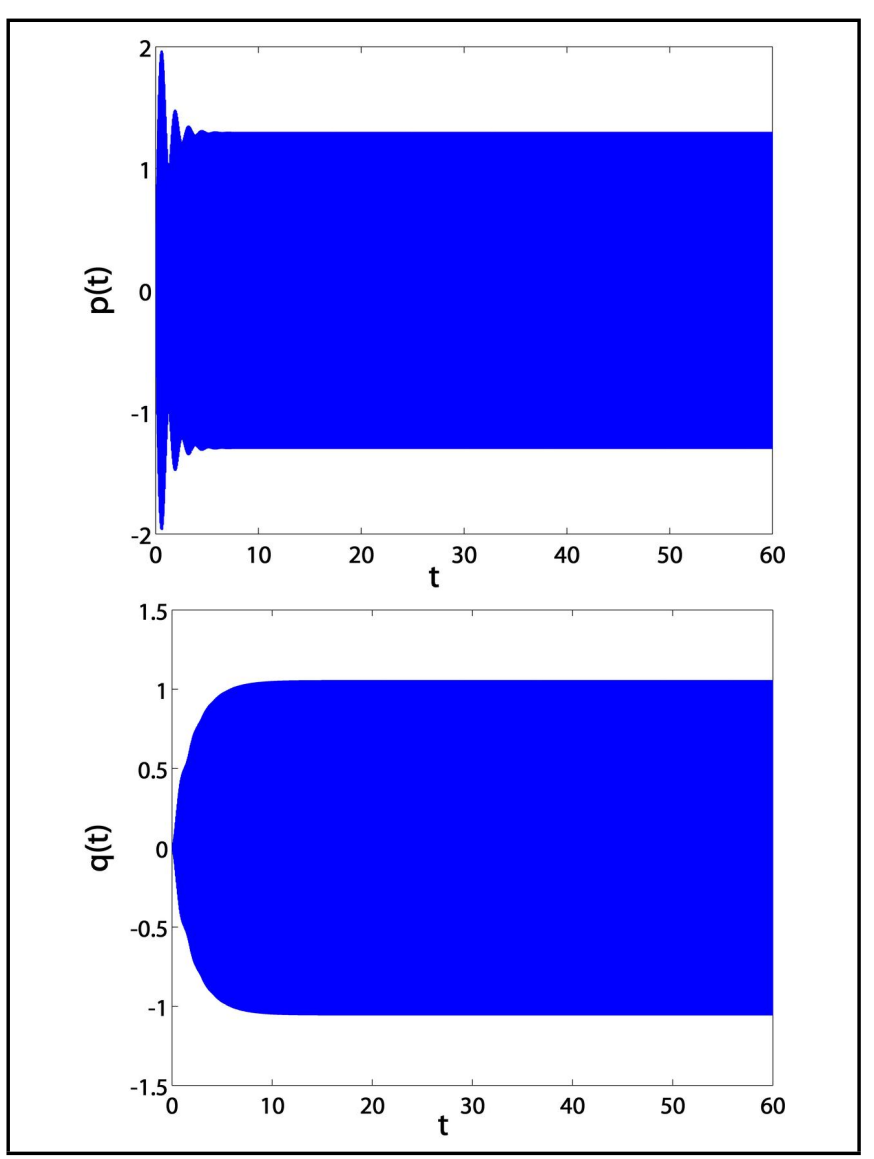

Figure 17. Time history after the control at zero initial conditions

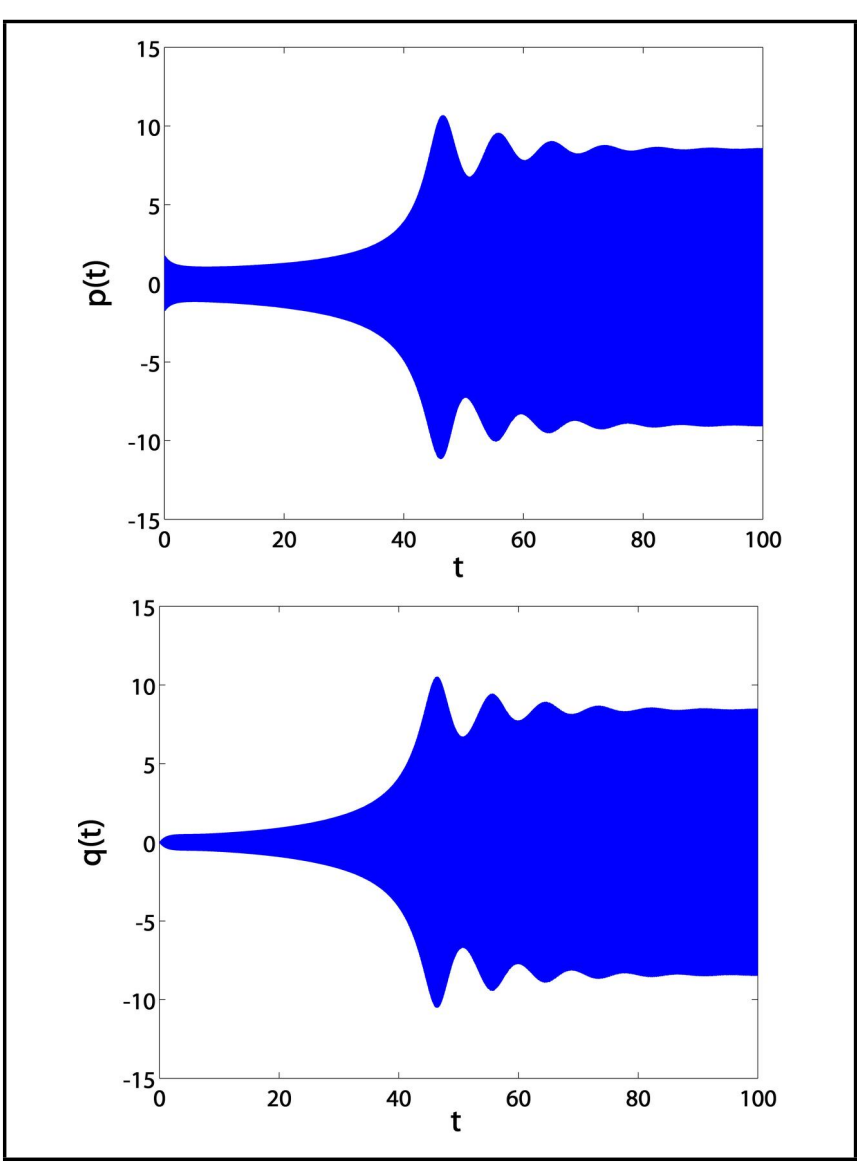

Figure 18. Time history before the control at zero initial conditions

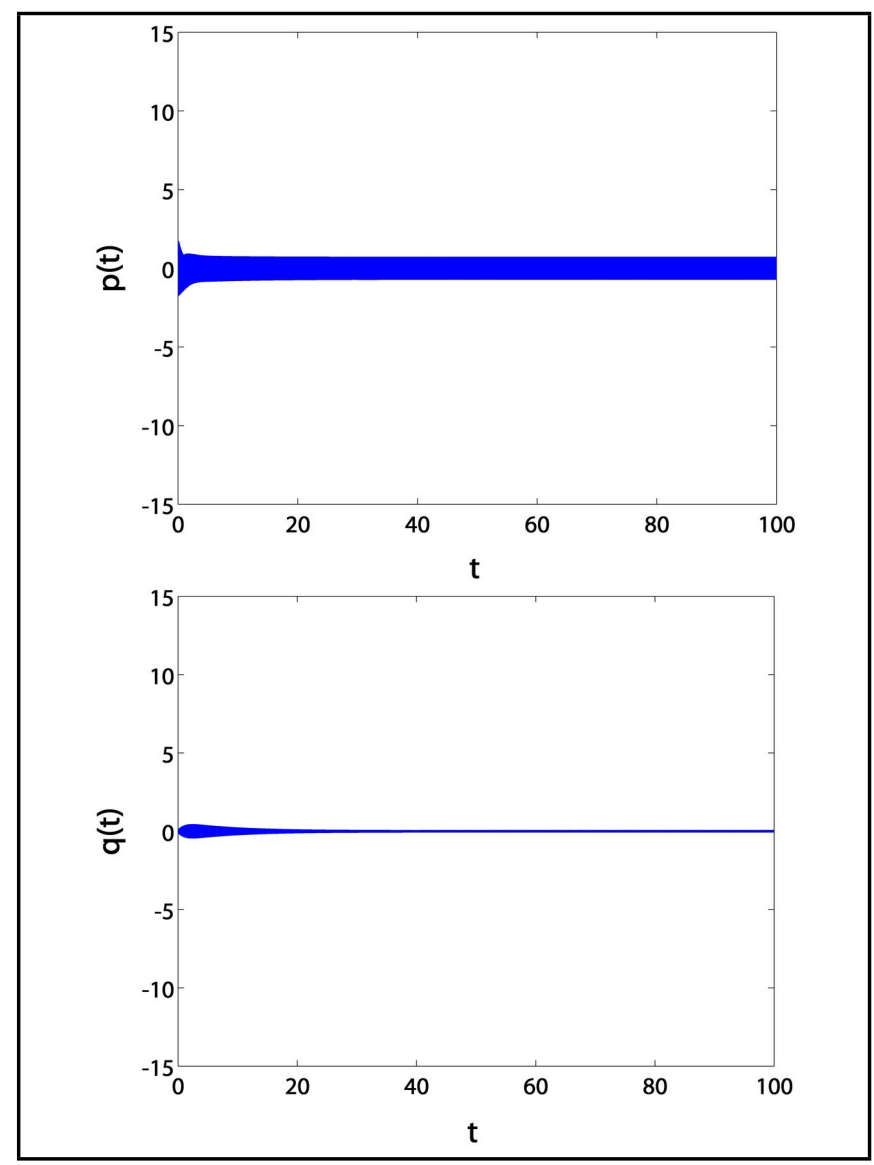

Figure 19. Time history after the control at zero initial conditions 


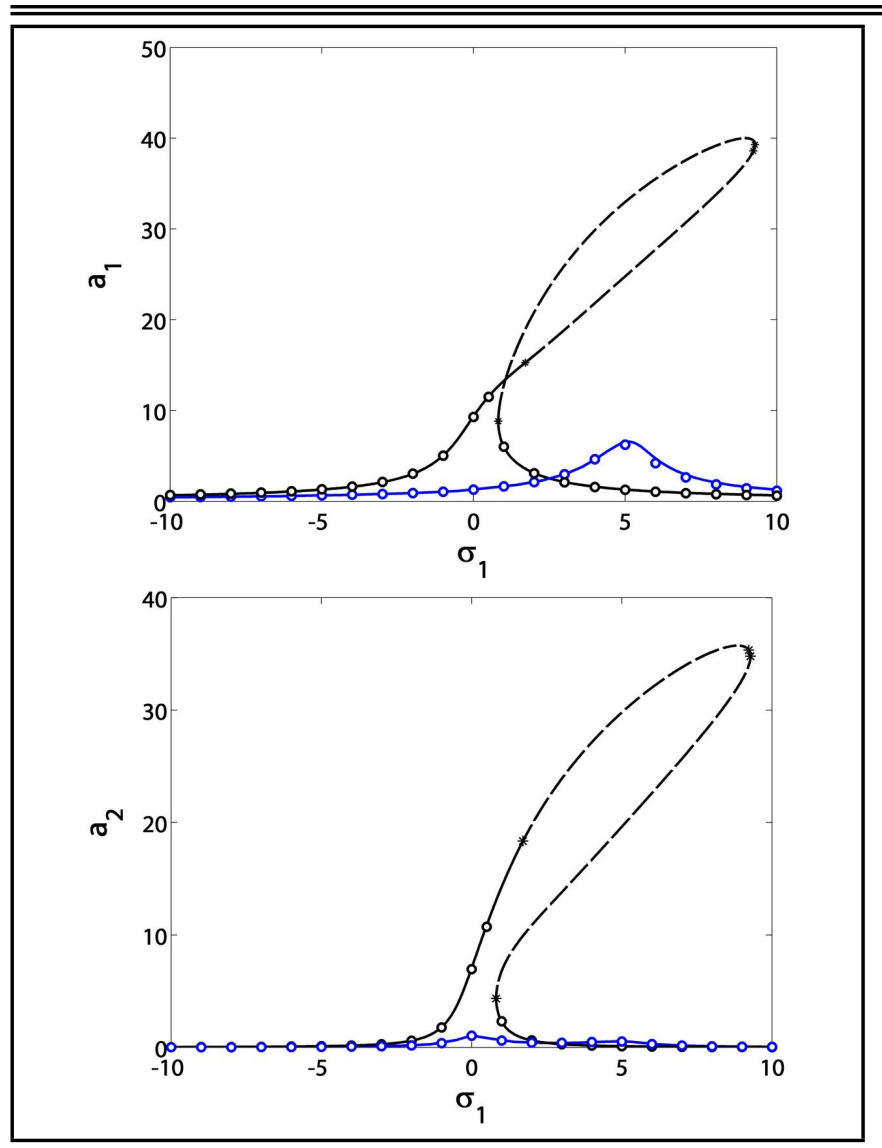

Figure 20. Verification of frequency response curves before and after the control (primary resonance)

lines refer to perturbation solutions, while the circles denote the ones resulted from the numerical integration. The following figures show the closeness between the numerical integration solutions and the approximate solutions before and after the control.

\section{CONCLUSIONS}

In this research, an active position and velocity feedback controller was applied to the compressor blade system, which was subjected to either primary excitation or principal parametric excitation. The approximate solutions of this combination were concluded using multiple time scales method. The Lyapunov first method was adopted to achieve a stability analysis for plotting the bifurcation diagrams. Eventually, the following can be concluded:

1. Primary resonance: The controller eliminated the saddle-node and Hopf bifurcation points and the curve had been suppressed to acceptable levels. Increasing $k_{1}$ and $k_{2}$ reduced the output amplitudes to achieve controller effectiveness $E_{a}$, about 7 and 6 for the first and second modes, respectively. The slope of the relation between the output amplitudes and excitation force decreased.

2. Principal parametric resonance: The controller eliminated the pitchfork bifurcation points and the system passed only through the trivial path. For $k_{1} \geq 175$ and $k_{2} \geq 1$, the trivial output could be guaranteed to achieve controller effectiveness $E_{a}$, about 13 and 2122 for the

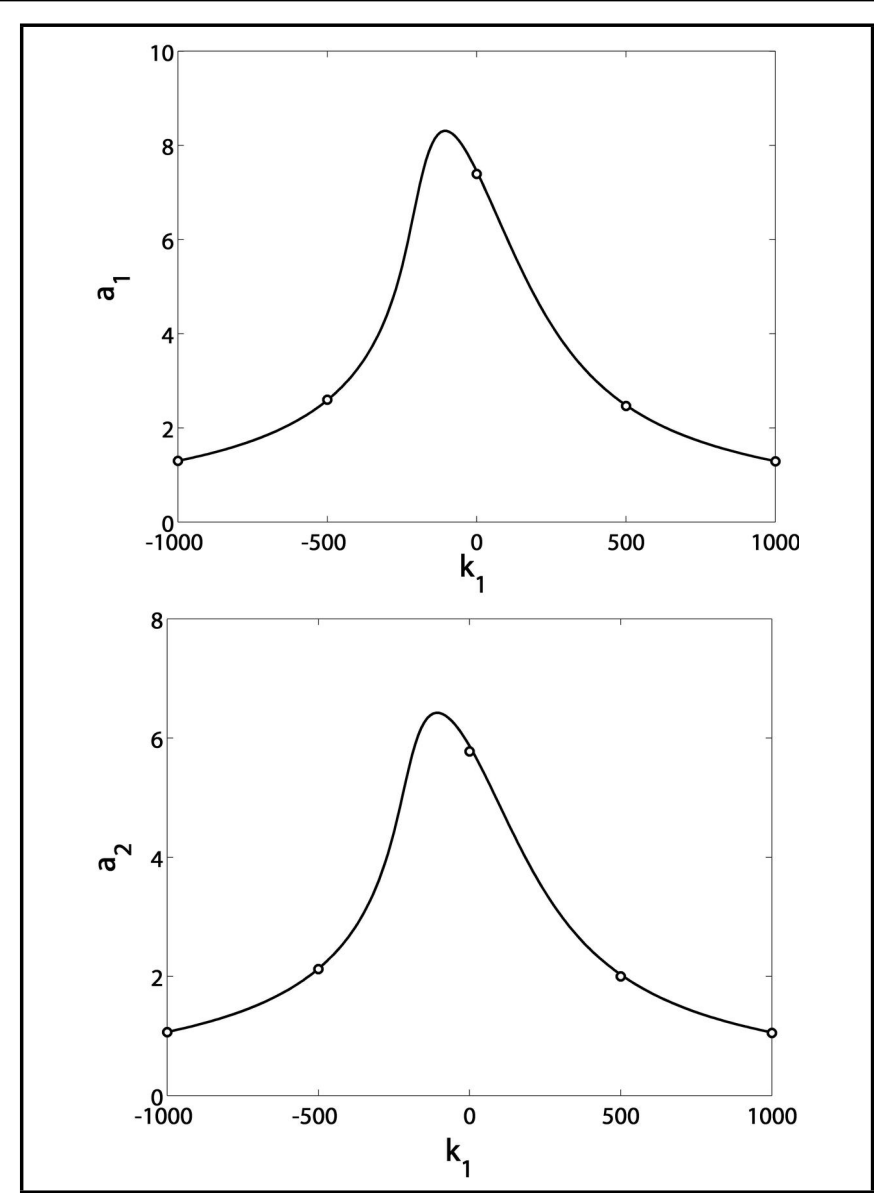

Figure 21. Verification of $k_{1}$ response curves (primary resonance)
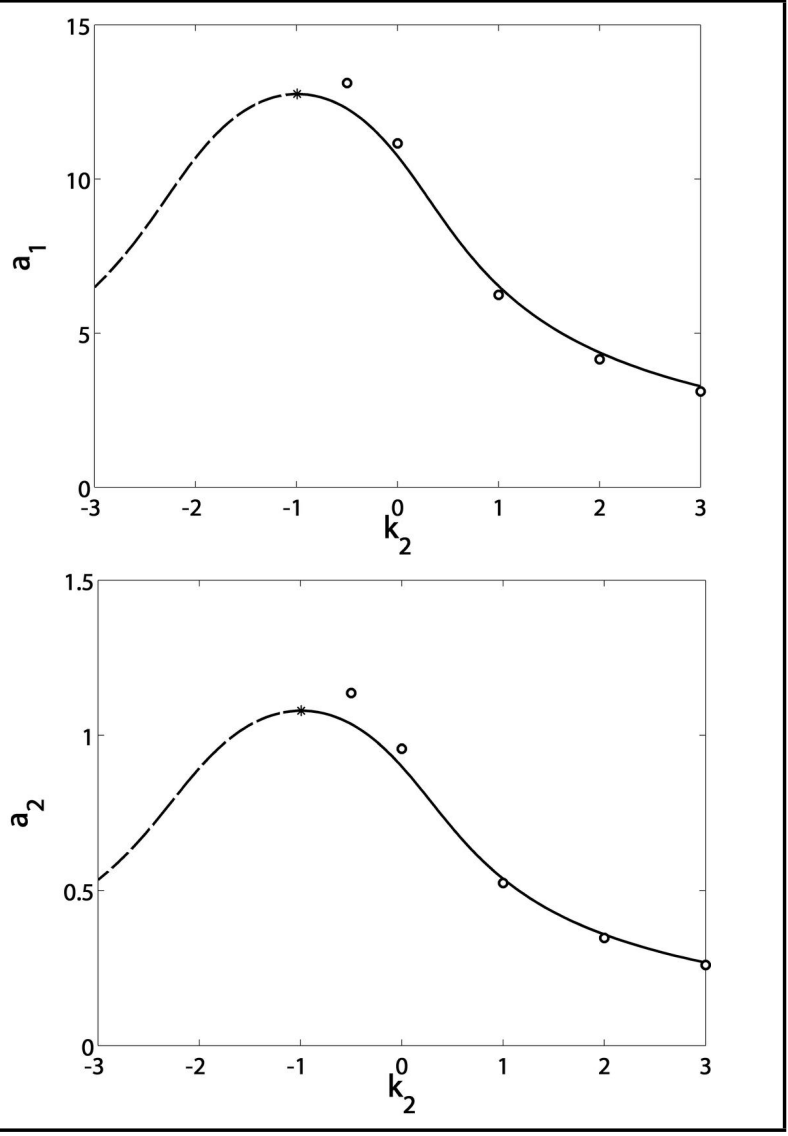

Figure 22. Verification of $k_{2}$ response curves (primary resonance) 


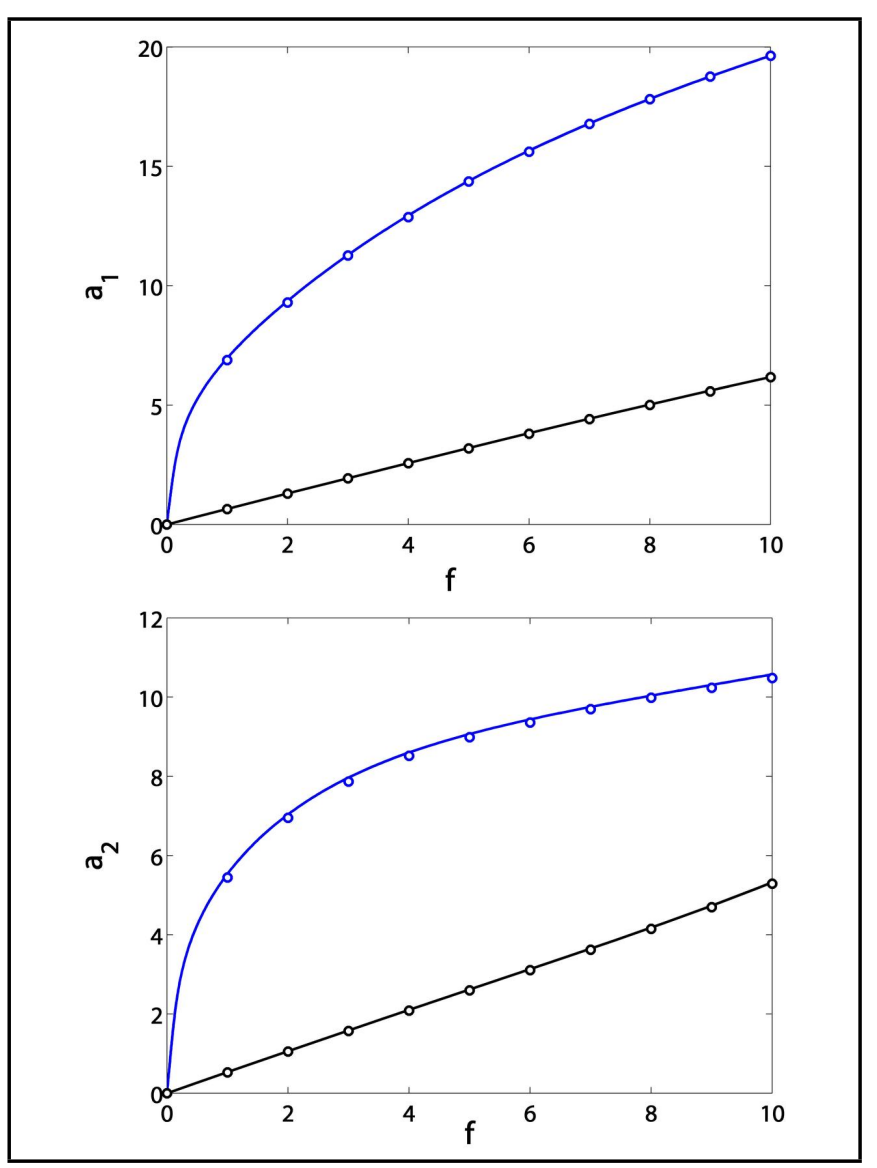

Figure 23. Verification of force response curves before and after the control (primary resonance)

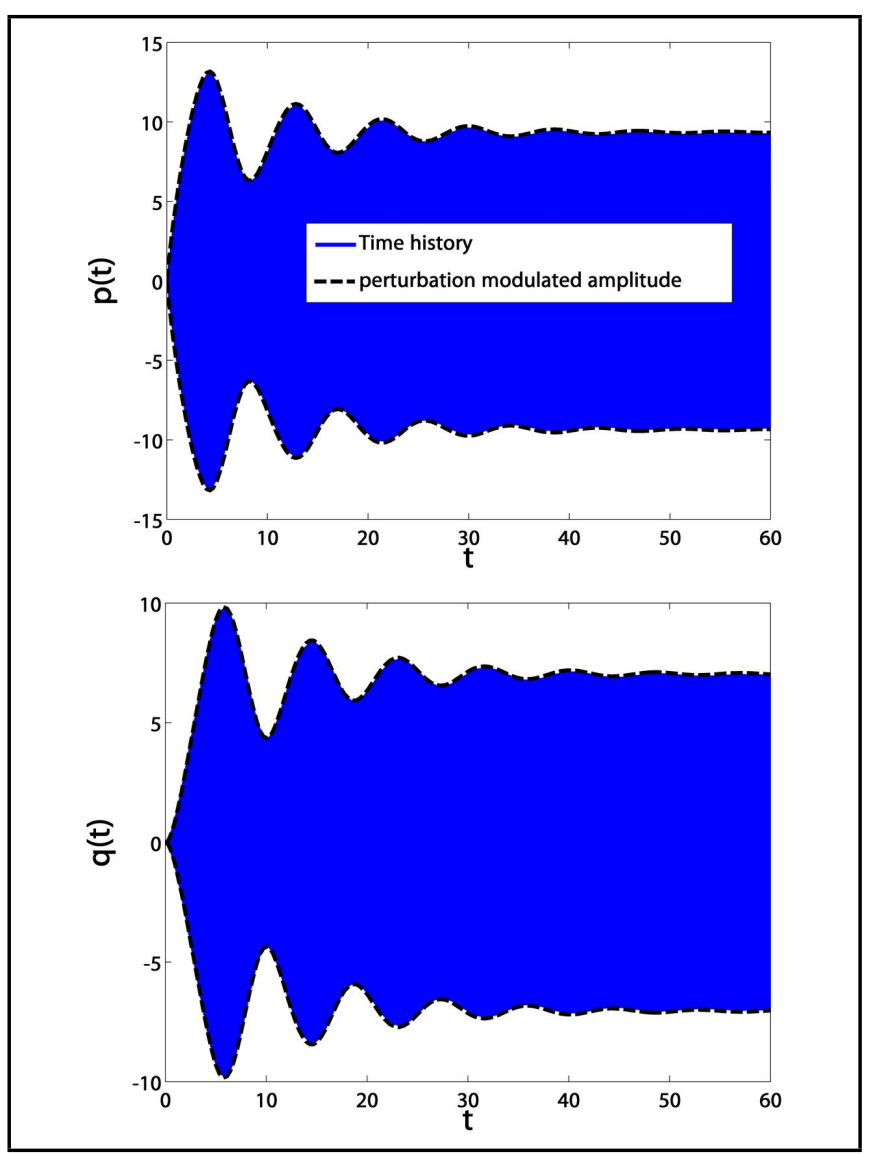

Figure 24. Verification of time history before the control (primary resonance)

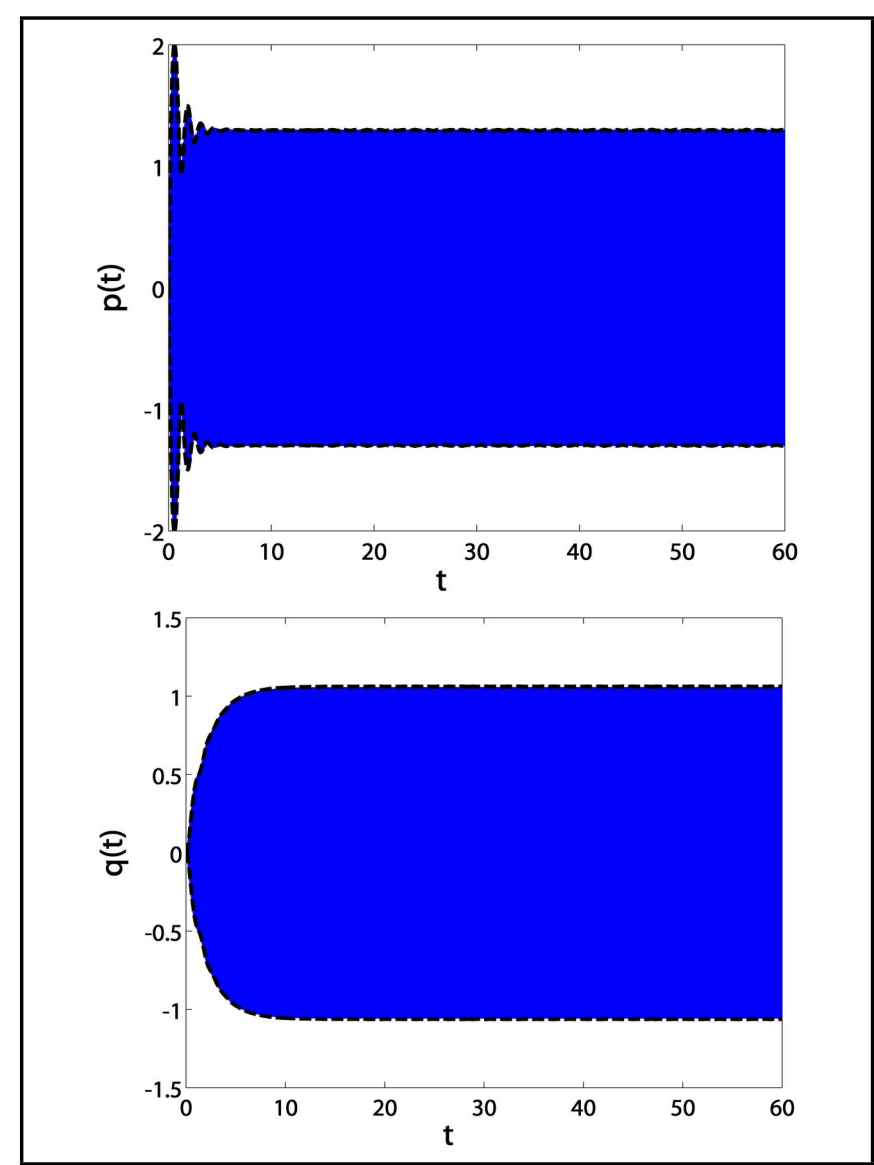

Figure 25. Verification of time history after the control (primary resonance)

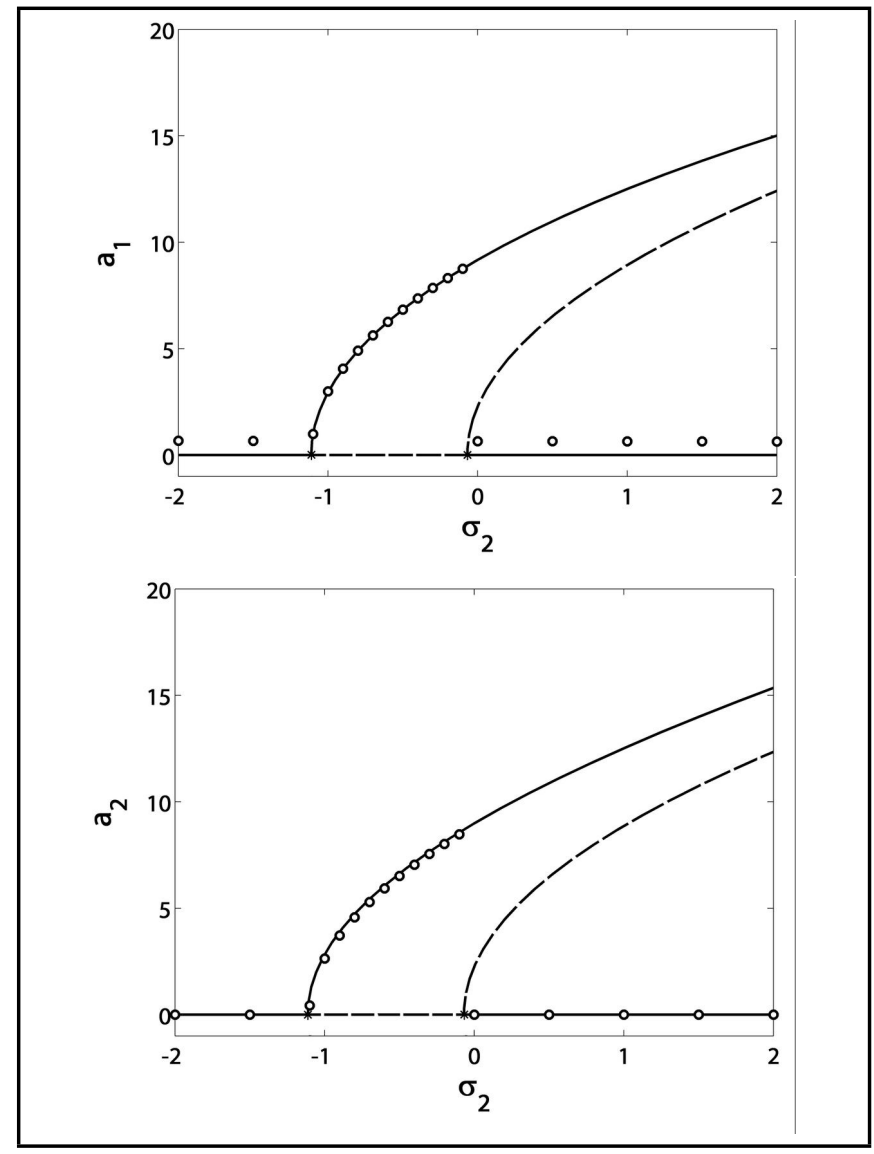

Figure 26. Verification of frequency response curves before the control (parametric resonance) 


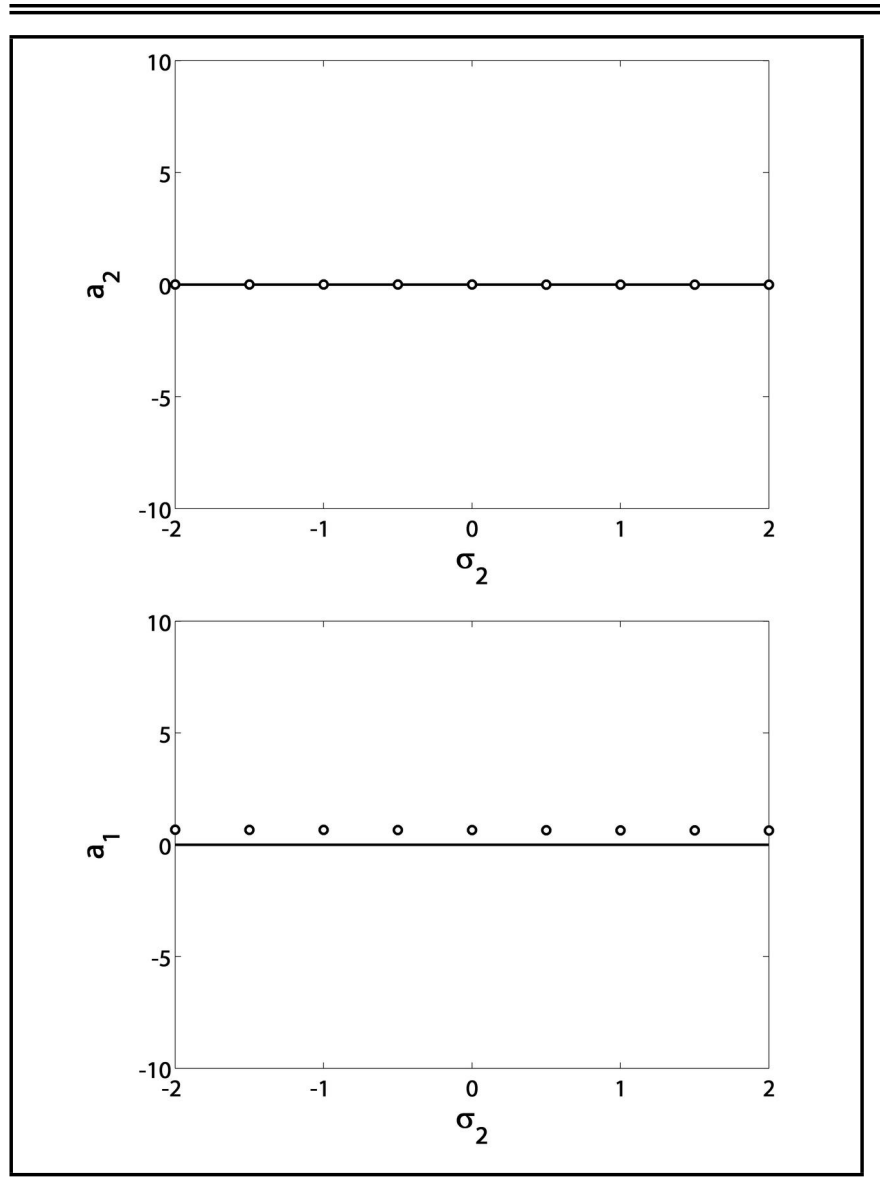

Figure 27. Verification of frequency response curves after the control (parametric resonance)

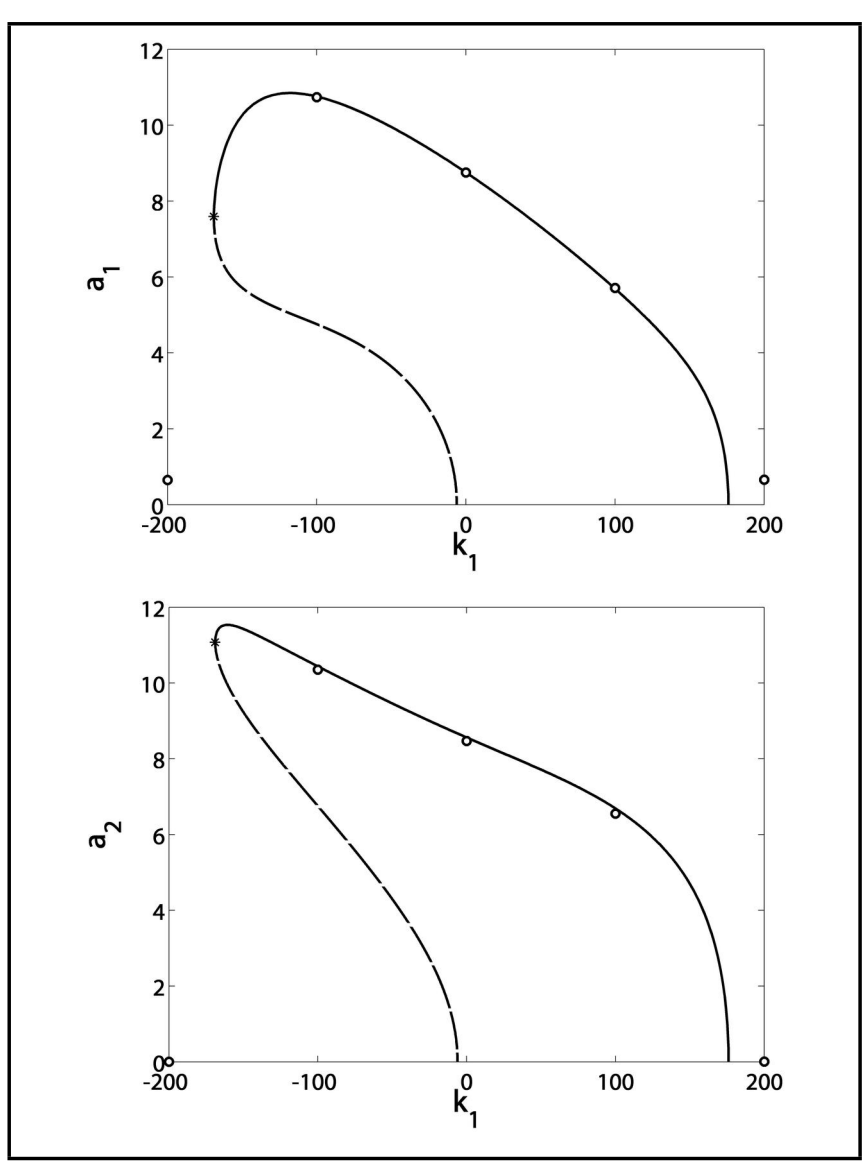

Figure 28. Verification of $k_{1}$ response curves (parametric resonance)
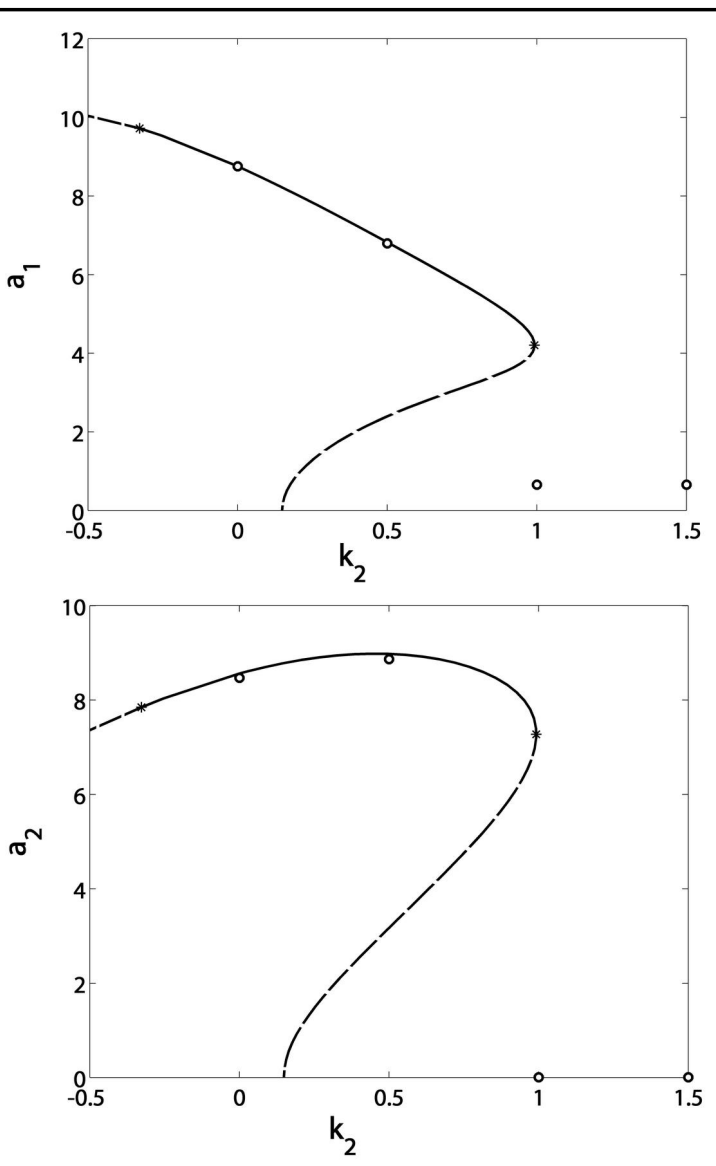

Figure 29. Verification of $k_{2}$ response curves (parametric resonance)

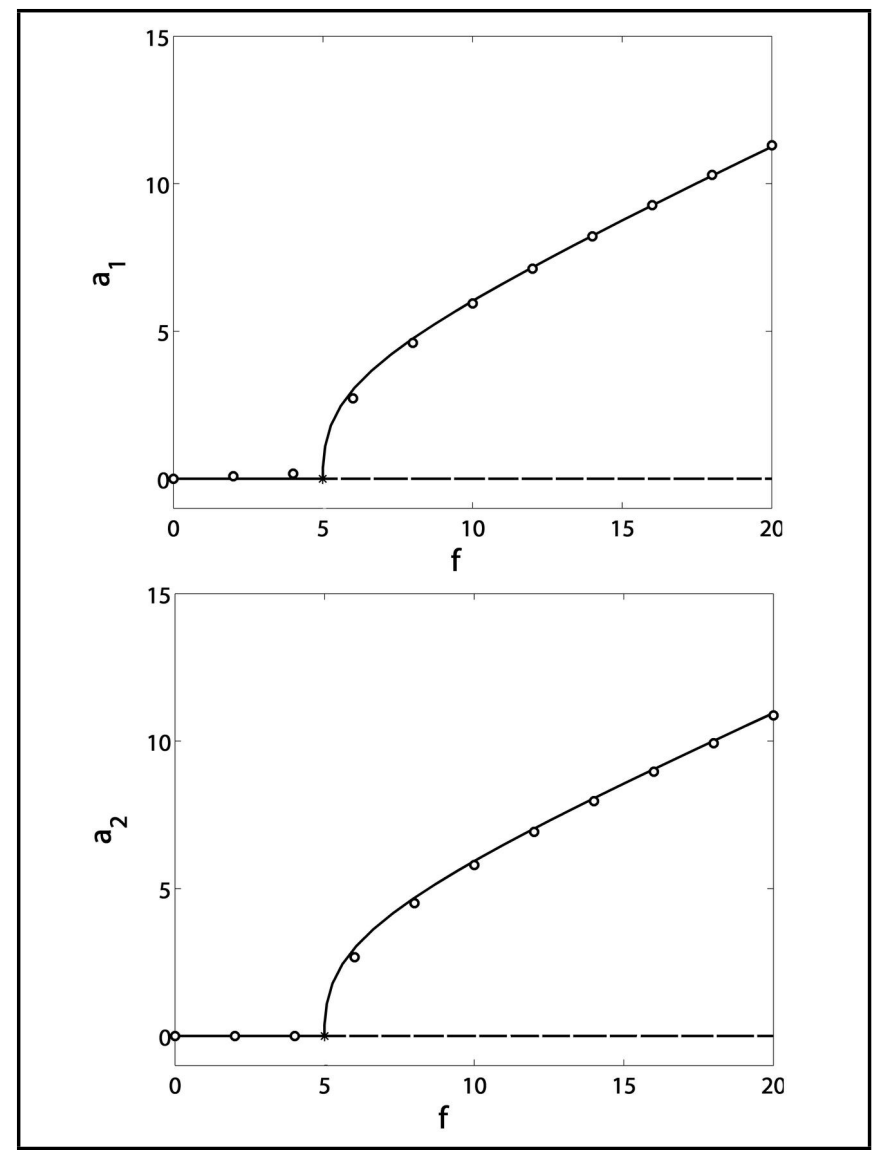

Figure 30. Verification of force response curves before the control (parametric resonance) 


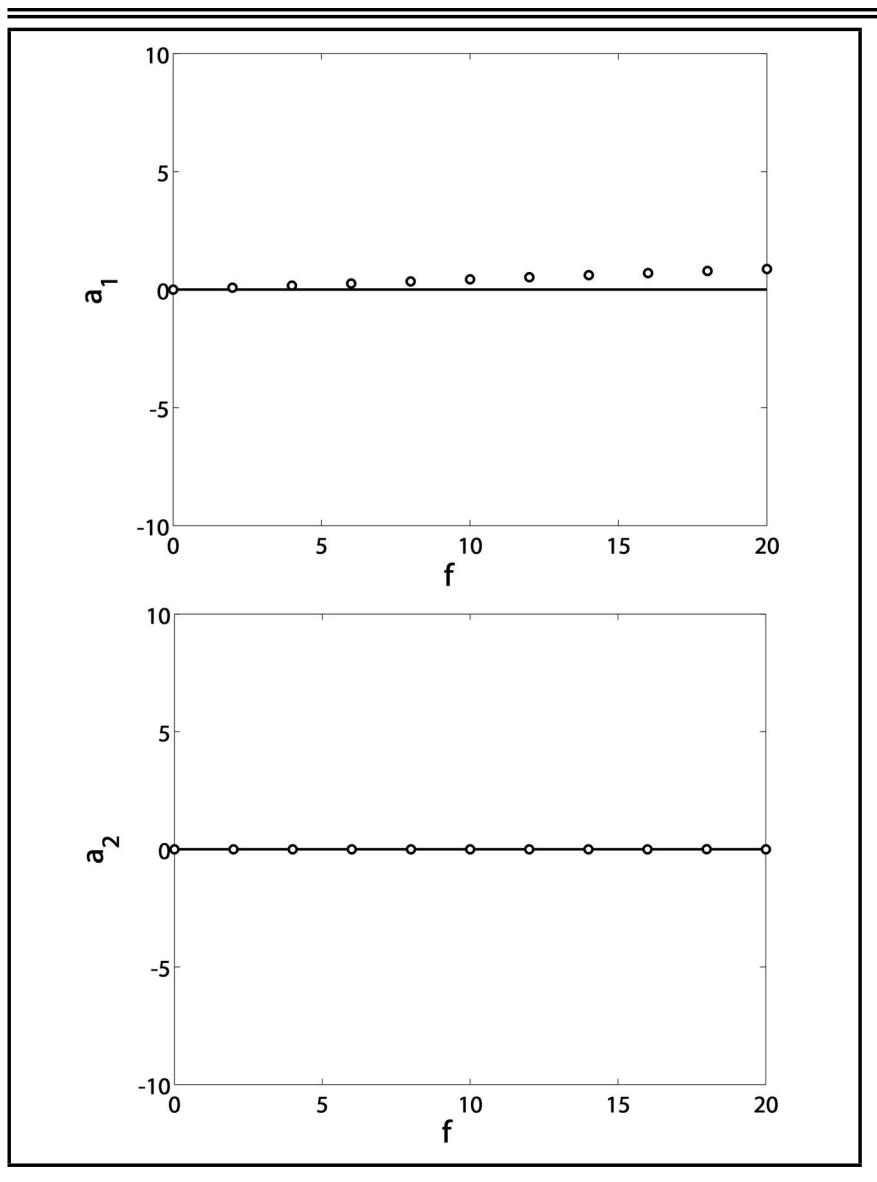

Figure 31. Verification of force response curves after the control (parametric resonance)

first and second modes, respectively. The force response curves had also changed after the control to pass through the trivial path only.

On the past work of controlling the compressor blade vibrations, Younesian and Esmailzadeh reduced the vibrations of the rotating beam using a time-increasing internal tensile force. $^{7}$ They showed that the suppression system could effectively reduce vibration by about $50 \%\left(E_{a}=2\right)$. In this paper, an active position and velocity feedback controller was applied to the compressor blade system subjected to either primary or parametric excitations. Upon the mentioned parameters in this paper, it was noticed that the controller effectiveness $E_{a}$ was about 7 and 6 for the first and second modes (primary), and was about 13 and 2122 for the first and second modes (parametric). This means that the controller reduced the vibrations of both modes to about $14 \%$ and $16 \%$ (primary), $7 \%$ and $0.05 \%$ (parametric).

\section{REFERENCES}

1 Yoo, H. H., Kwak, J. Y. and Chung, J. Vibration analysis of rotating pre-twisted blades with a concentrated mass, Journal of sound and vibration, 240 (5), 891-908, (2001). https://dx.doi.org/10.1006/jsvi.2000.3258

2 Sinha, S. K. Dynamic characteristics of a flexible bladed rotor with Coulomb damping due to tip-rub, Journal of sound vibration, 273 (4-5), 875-919, (2004). https://dx.doi.org/10.1016/s0022-460x(03)00647-3

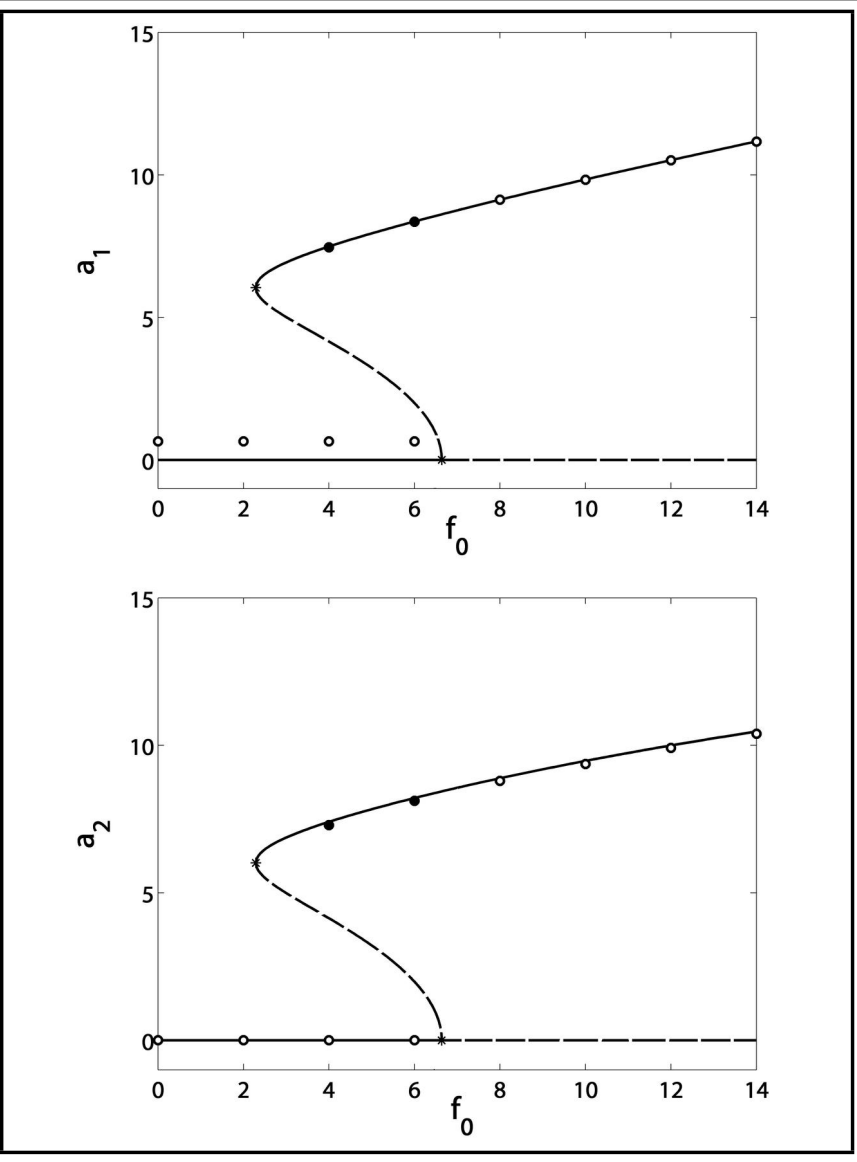

Figure 32. Verification of force response curves before the control (parametric resonance, the two black-filled circles are at initial conditions $p(0)=q(0)=$ 10)

3 Fazelzadeh, S. A., Malekzadeh, P., Zahedinejad, P. and Hosseini, M. Vibration analysis of functionally graded thin-walled rotating blades under high temperature supersonic flow using the differential quadrature method, Journal of sound vibration, 306 (1-2), 333-348, (2007). https://dx.doi.org/10.1016/j.jsv.2007.05.011

4 Yao, M. H., Chen, Y. P. and Zhang, W. Nonlinear vibrations of blade with varying rotating speed, Nonlinear Dynamics, 68 (4), 487-504, (2012). https://dx.doi.org/10.1007/s11071-011-0231-z

5 Yao, M. H., Zhang, W. and Chen, Y. P. Analysis on nonlinear oscillations and resonant responses of a compressor blade, Acta Mech, 225 (12), 3483-3510, (2014). https://dx.doi.org/10.1007/s00707-014-1151-z

6 Vadiraja, D. N. and Sahasrabudhe, A. D. Vibration analysis and optimal control of rotating pretwisted thin-walled beams using MFC actuators and sensors,Thin-Walled Structures, 47 (5), 555-567, (2009). https://dx.doi.org/10.1016/j.tws.2008.10.004

7 Younesian, D. and Esmailzadeh, E. Vibration suppression of rotating beams using time-varying internal tensile force, Journal of Sound and Vibration, 330 (2), 308-320, (2011). https://dx.doi.org/10.1016/j.jsv.2010.08.006

${ }^{8}$ Fey, R. H. B., Wouters, R. M. T. and Nijmeijer, H. Proportional and derivative control for steadystate vibration mitigation in a piecewise linear beam 


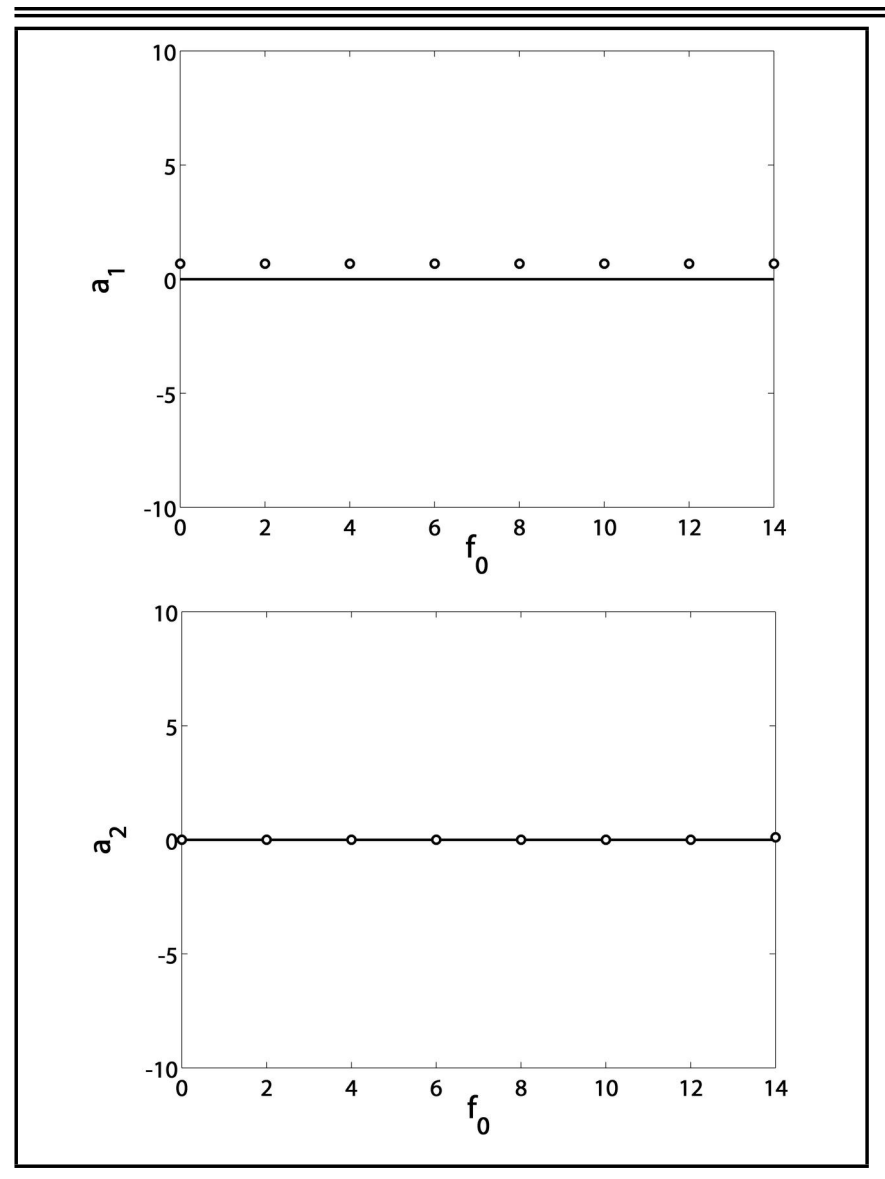

Figure 33. Verification of force response curves after the control (parametric resonance)

system, Nonlinear Dyn, 60 (4), 535-549, (2010). https://dx.doi.org/10.1007/s11071-009-9613-x

9 Warminski, J., Bochenski, M., Jarzyna, W., Filipek, P. and Augustyniak, M. Active suppression of nonlinear composite beam vibrations by selected control algorithms, Commun Nonlinear Sci Numer Simulat, 16 (5), 2237-2248, (2011). https://dx.doi.org/10.1016/j.cnsns.2010.04.055

10 Muhammad, A. K., Okamoto, S. and Lee, J. H. Comparison of proportional-derivative and active-force controls on vibration of a flexible single-link manipulator using finite-element method, Artif Life Robotics, 19 (4), 375-381, (2014). https://dx.doi.org/10.1007/s10015-014-0186-5

11 Eissa, M., Kandil, A., Kamel, M. and El-Ganaini, W. A. On controlling the response of primary and parametric resonances of a nonlinear magnetic levitation system, Meccanica, 50 (1), 233-251, (2015). https://dx.doi.org/10.1007/s11012-014-0069-9

12 Eissa, M., Kandil, A., El-Ganaini, W. A. and Kamel, M. Analysis of a nonlinear magnetic levitation system vibrations controlled by a time-delayed proportional-derivative controller, Nonlinear Dyn, 79 (2), 1217-1233, (2015). https://dx.doi.org/10.1007/s11071-014-1738-x

13 Nayfeh, A. H. and Mook, D. T. Nonlinear Oscillations, Wiley, New York, USA (1995). https://dx.doi.org/10.1002/9783527617586

\section{APPENDIX A}

$$
\begin{gathered}
v_{11}=\frac{\partial \dot{a}_{1}}{\partial a_{1}}=-\mu_{1}-\frac{\beta_{5}}{8 \omega} a_{2}^{2} \sin \left(2 \phi_{2}\right)+\frac{\beta_{14} f^{2}}{8 \omega} \sin \left(2 \phi_{1}\right)-\frac{k_{2}}{2}, \\
v_{12}=\frac{\partial \dot{a}_{1}}{\partial \phi_{1}}=\frac{\beta_{14} f^{2}}{4 \omega} a_{1} \cos \left(2 \phi_{1}\right)+\frac{\beta_{16} \Omega f}{2 \omega} \sin \phi_{1}, \\
v_{13}=\frac{\partial \dot{a}_{1}}{\partial a_{2}}=-\frac{\beta_{13}}{2} \cos \phi_{2}-\frac{\beta_{11}}{2 \omega} \sin \phi_{2}-\frac{\beta_{5}}{4 \omega} a_{1} a_{2} \sin \left(2 \phi_{2}\right), \\
v_{14}=\frac{\partial \dot{a}_{1}}{\partial \phi_{2}}=\frac{\beta_{13}}{2} a_{2} \sin \phi_{2}-\frac{\beta_{11}}{2 \omega} a_{2} \cos \phi_{2}-\frac{\beta_{5}}{4 \omega} a_{1} a_{2}^{2} \cos \left(2 \phi_{2}\right), \\
v_{21}=\frac{\partial \dot{\phi}_{1}}{\partial a_{1}}=-\frac{\beta_{13}}{2} \frac{a_{2}}{a_{1}^{2}} \sin \phi_{2}+\frac{\beta_{11}}{2 \omega} \frac{a_{2}}{a_{1}^{2}} \cos \phi_{2}-\frac{3 \beta_{5}}{4 \omega} a_{1}- \\
\frac{\beta_{16} \Omega f}{2 \omega} \frac{1}{a_{1}^{2}} \sin \phi_{1}, \\
v_{23}=\frac{\partial \dot{\phi}_{1}}{\partial a_{2}}=\frac{\beta_{13}}{2} \frac{1}{a_{1}} \sin \phi_{2}-\frac{\beta_{11}}{2 \omega} \frac{1}{a_{1}} \cos \phi_{2}-\frac{\beta_{5}}{2 \omega} a_{2}- \\
v_{22}=\frac{\beta_{14} f^{2}}{4 \omega} \sin \left(2 \phi_{1}\right)+\frac{\beta_{16} \Omega f}{2 \omega} \frac{1}{a_{1}} \cos \phi_{1},
\end{gathered}
$$

$v_{24}=\frac{\partial \dot{\phi}_{1}}{\partial \phi_{2}}=\frac{\beta_{13}}{2} \frac{a_{2}}{a_{1}} \cos \phi_{2}+\frac{\beta_{11}}{2 \omega} \frac{a_{2}}{a_{1}} \sin \phi_{2}+\frac{\beta_{5}}{4 \omega} a_{2}^{2} \sin \left(2 \phi_{2}\right)$

$v_{31}=\frac{\partial \dot{a}_{2}}{\partial a_{1}}=-\frac{\beta_{22}}{2} \cos \phi_{2}+\frac{\beta_{21}}{2 \omega} \sin \phi_{2}+\frac{\beta_{5}}{4 \omega} a_{1} a_{2} \sin \left(2 \phi_{2}\right)$,

$$
v_{32}=\frac{\partial \dot{a}_{2}}{\partial \phi_{1}}=\frac{\beta_{24} f^{2}}{4 \omega} a_{2} \cos \left(2 \phi_{1}-2 \phi_{2}\right),
$$

$$
\begin{gathered}
v_{33}=\frac{\partial \dot{a}_{2}}{\partial a_{2}}=-\mu_{2}+\frac{\beta_{5}}{8 \omega} a_{1}^{2} \sin \left(2 \phi_{2}\right)+\frac{\beta_{24} f^{2}}{8 \omega} \sin \left(2 \phi_{1}-2 \phi_{2}\right), \\
v_{34}=\frac{\partial \dot{a}_{2}}{\partial \phi_{2}}=\frac{\beta_{22}}{2} a_{1} \sin \phi_{2}+\frac{\beta_{21}}{2 \omega} a_{1} \cos \phi_{2}+ \\
\frac{\beta_{5}}{4 \omega} a_{1}^{2} a_{2} \cos \left(2 \phi_{2}\right)-\frac{\beta_{24} f^{2}}{4 \omega} a_{2} \cos \left(2 \phi_{1}-2 \phi_{2}\right), \\
v_{41}=\frac{\partial \dot{\phi}_{2}}{\partial a_{1}}=\frac{\beta_{22}}{2} \frac{1}{a_{2}} \sin \phi_{2}+\frac{\beta_{21}}{2 \omega} \frac{1}{a_{2}} \cos \phi_{2}+\frac{\beta_{5}}{2 \omega} a_{1}+ \\
\frac{\beta_{5}}{4 \omega} a_{1} \cos \left(2 \phi_{2}\right)-\frac{3 \beta_{5}}{4 \omega} a_{1}-\frac{\beta_{13}}{2} \frac{a_{2}}{a_{1}^{2}} \sin \phi_{2}+ \\
\frac{\beta_{11}}{2 \omega} \frac{a_{2}}{a_{1}^{2}} \cos \phi_{2}-\frac{\beta_{16} \Omega f}{2 \omega} \frac{1}{a_{1}^{2}} \sin \phi_{1},
\end{gathered}
$$




$$
\begin{aligned}
& v_{42}=\frac{\partial \dot{\phi}_{2}}{\partial \phi_{1}}=\frac{\beta_{24} f^{2}}{4 \omega} \sin \left(2 \phi_{1}-2 \phi_{2}\right)-\frac{\beta_{14} f^{2}}{4 \omega} \sin \left(2 \phi_{1}\right)+ \\
& \frac{\beta_{16} \Omega f}{2 \omega} \frac{1}{a_{1}} \cos \phi_{1} \\
& v_{43}=\frac{\partial \dot{\phi}_{2}}{\partial a_{2}}=-\frac{\beta_{22}}{2} \frac{a_{1}}{a_{2}^{2}} \sin \phi_{2}-\frac{\beta_{21}}{2 \omega} \frac{a_{1}}{a_{2}^{2}} \cos \phi_{2}-\frac{\beta_{5}}{2 \omega} a_{2}- \\
& \frac{\beta_{5}}{4 \omega} a_{2} \cos \left(2 \phi_{2}\right)+\frac{3 \beta_{5}}{4 \omega} a_{2}+\frac{\beta_{13}}{2} \frac{1}{a_{1}} \sin \phi_{2}-\frac{\beta_{11}}{2 \omega} \frac{1}{a_{1}} \cos \phi_{2}, \\
& v_{44}=\frac{\partial \dot{\phi}_{2}}{\partial \phi_{2}}=\frac{\beta_{22}}{2} \frac{a_{1}}{a_{2}} \cos \phi_{2}-\frac{\beta_{21}}{2 \omega} \frac{a_{1}}{a_{2}} \sin \phi_{2}- \\
& \frac{\beta_{5}}{4 \omega} a_{1}^{2} \sin \left(2 \phi_{2}\right)-\frac{\beta_{24} f^{2}}{4 \omega} \sin \left(2 \phi_{1}-2 \phi_{2}\right)+ \\
& \frac{\beta_{13}}{2} \frac{a_{2}}{a_{1}} \cos \phi_{2}+\frac{\beta_{11}}{2 \omega} \frac{a_{2}}{a_{1}} \sin \phi_{2}+\frac{\beta_{5}}{4 \omega} a_{2}^{2} \sin \left(2 \phi_{2}\right), \\
& \delta_{11}=\frac{\partial \dot{a}_{1}}{\partial a_{1}}=-\mu_{1}-\frac{\beta_{5}}{8 \omega} a_{2}^{2} \sin \left(2 \phi_{2}\right)+\frac{f_{0} f \beta_{14}}{2 \omega} \sin \phi_{1}-\frac{k_{2}}{2}, \\
& \delta_{12}=\frac{\partial \dot{a}_{1}}{\partial \phi_{1}}=\frac{f_{0} f \beta_{14}}{2 \omega} a_{1} \cos \phi_{1} \\
& \delta_{13}=\frac{\partial \dot{a}_{1}}{\partial a_{2}}=-\frac{\beta_{13}}{2} \cos \phi_{2}-\frac{\beta_{11}}{2 \omega} \sin \phi_{2}-\frac{\beta_{5}}{4 \omega} a_{1} a_{2} \sin \left(2 \phi_{2}\right), \\
& \delta_{14}=\frac{\partial \dot{a}_{1}}{\partial \phi_{2}}=\frac{\beta_{13}}{2} a_{2} \sin \phi_{2}-\frac{\beta_{11}}{2 \omega} a_{2} \cos \phi_{2}-\frac{\beta_{5}}{4 \omega} a_{1} a_{2}^{2} \cos \left(2 \phi_{2}\right), \\
& \delta_{33}=\frac{\partial \dot{a}_{2}}{\partial a_{2}}=-\mu_{2}+\frac{\beta_{5}}{8 \omega} a_{1}^{2} \sin \left(2 \phi_{2}\right)+\frac{f_{0} f \beta_{24}}{2 \omega} \sin \left(\phi_{1}-2 \phi_{2}\right), \\
& \delta_{21}=\frac{\partial \dot{\phi}_{1}}{\partial a_{1}}=-\beta_{13} \frac{a_{2}}{a_{1}^{2}} \sin \phi_{2}+\frac{\beta_{11}}{\omega} \frac{a_{2}}{a_{1}^{2}} \cos \phi_{2}-\frac{3 \beta_{5}}{2 \omega} a_{1} \\
& \delta_{22}=\frac{\partial \dot{\phi}_{1}}{\partial \phi_{1}}=-\frac{f_{0} f \beta_{14}}{\omega} \sin \phi_{1} \\
& \delta_{23}=\frac{\partial \dot{\phi}_{1}}{\partial a_{2}}=\beta_{13} \frac{1}{a_{1}} \sin \phi_{2}-\frac{\beta_{11}}{\omega} \frac{1}{a_{1}} \cos \phi_{2}- \\
& \frac{\beta_{5}}{\omega} a_{2}-\frac{\beta_{5}}{2 \omega} a_{2} \cos \left(2 \phi_{2}\right)
\end{aligned}
$$$$
\delta_{34}=\frac{\partial \dot{a}_{2}}{\partial \phi_{2}}=\frac{\beta_{22}}{2} a_{1} \sin \phi_{2}+\frac{\beta_{21}}{2 \omega} a_{1} \cos \phi_{2}+
$$$$
\frac{\beta_{5}}{4 \omega} a_{1}^{2} a_{2} \cos \left(2 \phi_{2}\right)-\frac{f_{0} f \beta_{24}}{\omega} a_{2} \cos \left(\phi_{1}-2 \phi_{2}\right),
$$$$
\delta_{41}=\frac{\partial \dot{\phi}_{2}}{\partial a_{1}}=\frac{\beta_{22}}{2} \frac{1}{a_{2}} \sin \phi_{2}+\frac{\beta_{21}}{2 \omega} \frac{1}{a_{2}} \cos \phi_{2}+\frac{\beta_{5}}{2 \omega} a_{1}+
$$$$
\frac{\beta_{5}}{4 \omega} a_{1} \cos \left(2 \phi_{2}\right)-\frac{\beta_{13}}{2} \frac{a_{2}}{a_{1}^{2}} \sin \phi_{2}+\frac{\beta_{11}}{2 \omega} \frac{a_{2}}{a_{1}^{2}} \cos \phi_{2}-\frac{3 \beta_{5}}{4 \omega} a_{1},
$$$$
\delta_{42}=\frac{\partial \dot{\phi}_{2}}{\partial \phi_{1}}=\frac{f_{0} f \beta_{24}}{2 \omega} \sin \left(\phi_{1}-2 \phi_{2}\right)-\frac{f_{0} f \beta_{14}}{2 \omega} \sin \phi_{1}
$$$$
\delta_{43}=\frac{\partial \dot{\phi}_{2}}{\partial a_{2}}=-\frac{\beta_{22}}{2} \frac{a_{1}}{a_{2}^{2}} \sin \phi_{2}-\frac{\beta_{21}}{2 \omega} \frac{a_{1}}{a_{2}^{2}} \cos \phi_{2}+\frac{3 \beta_{5}}{4 \omega} a_{2}+
$$$$
\frac{\beta_{13}}{2} \frac{1}{a_{1}} \sin \phi_{2}-\frac{\beta_{11}}{2 \omega} \frac{1}{a_{1}} \cos \phi_{2}-\frac{\beta_{5}}{2 \omega} a_{2}-\frac{\beta_{5}}{4 \omega} a_{2} \cos \left(2 \phi_{2}\right),
$$

\section{APPENDIX B}

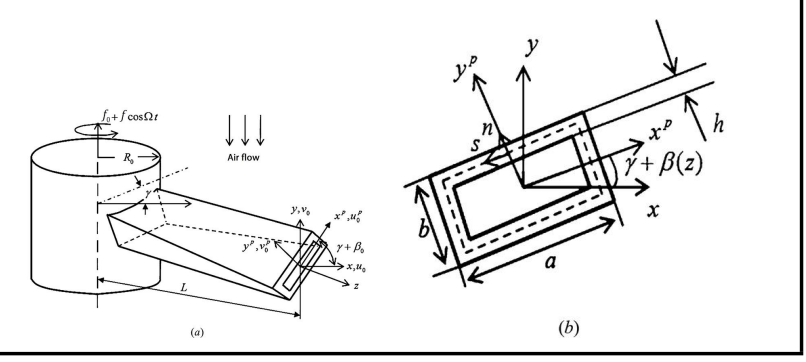

$\delta_{24}=\frac{\partial \dot{\phi}_{1}}{\partial \phi_{2}}=\beta_{13} \frac{a_{2}}{a_{1}} \cos \phi_{2}+\frac{\beta_{11}}{\omega} \frac{a_{2}}{a_{1}} \sin \phi_{2}+\frac{\beta_{5}}{2 \omega} a_{2}^{2} \sin \left(2 \phi_{2}\right)$,

Figure 34. Rotating compressor blade model, (a) pre-twisted thin-walled blade, and (b) blade cross section

The following derivation was explained in-detail in Yao et al. ${ }^{4,5}$ As in Fig. 34, consider a pre-twisted flexible cantilever blade with length $L$ mounted on a rigid hub with radius $R_{0}$. The blade rotates at a varying rotating speed about its polar axis, as shown in Fig. 34a. It is assumed that the rotating speed is represented as a periodic rotating speed $f \cos \Omega t$ on a steady 
state rotating speed $f_{0}$, namely, $F=f_{0}+f \cos \Omega t$. The blade is allowed to vibrate flexurally in the plane that makes an angle $\gamma$, which is considered to be the setting angle with the plane of rotation. The rotating blade is treated as a pre-twist, presetting, thin-walled rotating cantilever beam. The thickness of the thin-walled beam is $h$, and the length and the width of the cross section of the beam are $a$ and $b$, respectively. To derive the analytical model of the thin-walled beam, the following assumptions are made:

- The shape of the cross section and all of its geometrical dimensions remain invariant in its plane. Cross sections of the beam are assumed rigid in their own planes.

- Compared to the unity, the ratio $\frac{h}{r}$ is negligibly small. The wall thickness of the beam is $h$, and the radius of curvature is $r$ at any point of the beam wall.

- Considering that the thin beam model is adopted in this paper, the transverse shear effect is neglected.

- The axial displacement $w$ is much smaller than $u$ or $v$ so that the derivatives of $w$ can be neglected in the straindisplacement relations.

The origin of the rotating coordinate systems $(x, y, z)$ is located at the blade root. The local coordinates $\left(x^{p}, y^{p}, z^{p}\right)$ are defined, where $x^{p}$ and $y^{p}$ are the principal axes of an arbitrary beam cross section as shown in Fig. 34b. The transformation formulas between two coordinate systems $(x, y, z)$ and $\left(x^{p}, y^{p}, z^{p}\right)$ are presented as follows:

$$
\begin{gathered}
x=x^{p} \cos (\gamma+\beta(z))-y^{p} \sin (\gamma+\beta(z)), \\
y=x^{p} \sin (\gamma+\beta(z))+y^{p} \cos (\gamma+\beta(z)), \\
z=z^{p},
\end{gathered}
$$

where $\beta(z)=\frac{\beta_{0} z}{L}$ denotes the pre-twist angle of a current beam cross section, and $\beta_{0}$ is the pre-twist at the beam tip.

The Hamilton principle is employed to derive the equation of motion for the rotating blade. The Hamilton principle is stated as

$$
\int_{0}^{t}(\delta K-\delta U+\delta W) d t=0,
$$

where $K$ and $U$, respectively, denote the kinetic energy and the strain energy, $W$ is the virtual work of external forces, $t$ denotes time, and $\delta$ is the variation operator. Yao et al. have done detailed mathematical derivations to get the non-dimensional governing equations of the nonlinear vibration for the rotating blade as follows: ${ }^{4,5}$

$$
\begin{gathered}
\ddot{u}_{0}-F^{2} u_{0}-F^{2}\left[R(z) u^{\prime \prime}{ }_{0}+R^{\prime}(z) u_{0}^{\prime}\right]+\alpha \Delta T u^{\prime \prime}{ }_{0}- \\
{\left[a_{3}(z) v^{\prime \prime}{ }_{0}-a_{6}(z) u_{0}^{\prime \prime}\right]^{\prime \prime}=u_{0}^{\prime}\left(u_{0}^{\prime} u^{\prime \prime}{ }_{0}+v^{\prime}{ }_{0} v_{0}\right)+} \\
u_{0}^{\prime \prime}\left[\frac{1}{2}\left(u_{0}^{\prime}\right)^{2}+\frac{1}{2}\left(v_{0}^{\prime}\right)^{2}\right]-\dot{F}\left(R_{0}+z\right)+p_{x}, \\
\ddot{v}_{0}-F^{2}\left[R(z) v_{0}^{\prime \prime}+R^{\prime}(z) v_{0}^{\prime}\right]+\alpha \Delta T v_{0}^{\prime \prime}- \\
{\left[a_{3}(z) u^{\prime \prime}{ }_{0}-a_{4}(z) v^{\prime \prime}{ }_{0}\right]^{\prime \prime}=v_{0}^{\prime}\left(u_{0}^{\prime} u^{\prime \prime}{ }_{0}+v_{0}^{\prime} v^{\prime \prime}{ }_{0}\right)+} \\
v_{0}^{\prime \prime}\left[\frac{1}{2}\left(u_{0}^{\prime}\right)^{2}+\frac{1}{2}\left(v_{0}^{\prime}\right)^{2}\right]+p_{y},
\end{gathered}
$$

where the dots and primes, respectively, represent partial differentiation with respect to $t$ and $z$. Galerkin's approach is used to truncate (25) to obtain a two-degree-of-freedom nonlinear ordinary system of differential equations under hightemperature supersonic gas flow and varying rotating speed. By the idea of a one-term Galerkin truncation, the modes of the rotating blade can be cast into the form:

$$
v_{0}=q(t) G(z) \quad \text { and } \quad v_{0}=q(t) G(z),
$$

where function $G(z)$ is a linear mode of the free vibration for the cantilever beam, which has the following form:

$$
\begin{gathered}
G(z)=\cosh (r z)-\cos (r z)- \\
{\left[\frac{\cosh (r)+\cos (r)}{\sinh (r)+\sin (r)}\right][\sinh (r z)-\sin (r z)],}
\end{gathered}
$$

where the coefficient $r$ is the root of $\cosh (r) \cos (r)+1=0$. The mode considered here is the typical form of the free vibration for the cantilever beam. Since the problem here is a weak nonlinear case, the mode of the free vibration can be considered as an approximation to the mode of the weak nonlinear system, and hence the convergence is guaranteed. Based on the experimented observations, the first-order mode makes the major contribution to the vibration responses of a dynamic system. Therefore, in order to qualitatively study the nonlinear behaviours of the rotating thin-walled blade, the first-order mode function is taken to discretize the governing equation by applying Galerkin's approach.

Substituting (26) and (27) into (25), multiplying (25) by $G(z)$ and integrating to $z$ from 0 to 1 , a two-degree-of-freedom nonlinear ordinary system of differential equations under hightemperature supersonic gas flow and varying rotating speed is obtained as:

$$
\begin{gathered}
\alpha_{5} \ddot{p}+\alpha_{11} \dot{p}+\alpha_{2} \dot{q}+\left(\alpha_{13}+\alpha_{17}-\alpha_{10} f_{0}^{2}\right) p+ \\
\left(\alpha_{14}+\alpha_{8}\right) q-2 \alpha_{10} p f_{0} f \cos (\Omega t)-\alpha_{10} p f^{2} \cos ^{2}(\Omega t) \\
+\alpha_{6} p^{3}+\alpha_{6} p q^{2}=\alpha_{9} \Omega f \sin (\Omega t), \\
\alpha_{5} \ddot{q}+\alpha_{21} \dot{q}+\alpha_{2} \dot{p}+\left(\alpha_{23}+\alpha_{8}\right) p+ \\
\left(\alpha_{24}+\alpha_{27}-\alpha_{20} f_{0}^{2}\right) q-2 \alpha_{20} q f_{0} f \cos (\Omega t)- \\
\alpha_{20} q f^{2} \cos ^{2}(\Omega t)+\alpha_{6} q^{3}+\alpha_{6} p^{2} q=0 .
\end{gathered}
$$

Dividing by $\alpha_{5}$ yields

$$
\begin{gathered}
\ddot{p}+2 \mu_{1} \dot{p}+\omega^{2} p+\beta_{13} \dot{q}+\beta_{11} q+\beta_{5} p q^{2}+\beta_{5} p^{3}= \\
2 f_{0} f \beta_{14} p \cos (\Omega t)+f^{2} \beta_{14} p \cos ^{2}(\Omega t)+f \beta_{16} \Omega \sin (\Omega t), \\
\ddot{q}+2 \mu_{2} \dot{q}+\omega^{2} q+\beta_{22} \dot{p}+\beta_{21} p+\beta_{5} p^{2} q+\beta_{5} q^{3}= \\
2 f_{0} f \beta_{24} q \cos (\Omega t)+f^{2} \beta_{24} q \cos ^{2}(\Omega t),
\end{gathered}
$$

where the coefficients of Eqs. 25, 28 and 29 are given in detail in refs. ${ }^{4,5}$ 